\title{
The vascular flora and vegetation of Queimada Grande Island, São Paulo State, southeastern Brazil
}

\author{
Bruno Coutinho Kurtz ${ }^{1 *}$, Vinicius Castro Souza ${ }^{2}$, Adriana Melo Magalhães ${ }^{3}$,Juliana de Paula-Souza ${ }^{4}$,
} Alexandre Romariz Duarte ${ }^{2}$ \& Gilberto Oliveira Joaquim-Jr. ${ }^{2,5}$

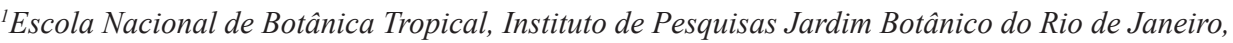
Rio de Janeiro, RJ, Brazil

${ }^{2}$ Universidade de São Paulo, Escola Superior de Agricultura Luiz de Queiroz, Departamento de Ciências Biológicas, Piracicaba, SP, Brazil

${ }^{3}$ Instituto Chico Mendes de Conservação da Biodiversidade, ARIE das Ilhas Queimada Pequena e Queimada Grande, Itanhaém, SP, Brazil

${ }^{4}$ Universidade Federal de São João del-Rei, Departamento de Ciências Exatas e Biológicas, Sete Lagoas, MG, Brazil ${ }^{5}$ Instituto Nacional de Metrologia Qualidade e Tecnologia, Rio de Janeiro, RJ, Brazil *Corresponding author: Bruno Coutinho Kurtz, e-mail: bkurtz@jbrj.gov.br

KURTZ, B. C., SOUZA, V. C., MAGALHÃES, A. M., PAUlA-SOUZA, J., DUARTE, A. R., JOAQUIM-JR., G. O. The vascular flora and vegetation of Queimada Grande Island, São Paulo State, southeastern Brazil. Biota Neotropica. 17(4): e20170336. http://dx.doi.org/10.1590/1676-0611-BN-2017-0336

\begin{abstract}
Studies of the vegetation on islands off the coast of southeastern Brazil are still very scarce, despite their importance for assessing, managing, and conserving insular biodiversity. We present here a list of the vascular flora of Queimada Grande Island (QGI; 24²9'10" S, 46 40'30" W, 57 ha, $33.2 \mathrm{~km}$ from the coast) in southeastern Brazil and describe its phytophysiognomies. The island is covered mainly by Atlantic Forest (Dense Ombrophilous Forest), as well as with rock outcrop and anthropogenic vegetation with herbaceous-shrub phytophysiognomies. QGI showed relatively low species richness $(\mathrm{S}=125)$ when compared to other Brazilian coastal islands. Herbaceous $(52)$ and climbing species (31) predominated on QGI. The richest families were Fabaceae (11 species), Poaceae (9), and Apocynaceae, Asteraceae and Orchidaceae ( 8 species each). Most species $(\mathrm{S}=112)$ are autochthonous from different phytophysiognomies of the southeastern Brazilian Atlantic Forest complex. Many species associated with anthropically disturbed areas $(\mathrm{S}=26)$ can be found on QGI, including the invasive grass Melinis minutiflora. There was a slight predominance of zoochory $(\mathrm{S}=50)$. We did not identify any species endemic to QGI. One of its species (Cattleya intermedia, Orchidaceae) is vulnerable at both national and regional levels, and another (Barrosoa apiculata, Asteraceae) is presumably extinct on the mainland in São Paulo State. The vascular flora of QGI originated from the mainland Atlantic Forest complex, following the pattern of other coastal islands in southeastern Brazil. The flora and vegetation of QGI reflect the combination of insular conditions, the small size of the island, habitat restriction, steep topography, incipient soils, and the past use of the area with the introduction of several foreign species. We recommend permanent monitoring of the vegetation cover of QGI and its management, in order to ensure the conservation of the local native biota.
\end{abstract}

Keywords: Atlantic Forest; biodiversity conservation; coastal islands; dispersal syndromes; invasive species; protected areas.

\section{Flora vascular e vegetação da Ilha Queimada Grande, São Paulo, sudeste do Brasil}

Resumo: Estudos sobre a vegetação de ilhas costeiras no sudeste do Brasil ainda são muito escassos, apesar de sua importância para a avaliação, manejo e conservação da biodiversidade insular. Nós apresentamos aqui uma lista da flora vascular da Ilha Queimada Grande (IQG; 2429'10" S, 46²40'30" W, 57 ha, 33,2 km da costa), sudeste do Brasil, e descrevemos suas fitofisionomias. A ilha é recoberta principalmente por Floresta Atlântica (Floresta Ombrófila Densa), bem como por vegetação sobre afloramento rochoso e vegetação antrópica com fisionomias herbáceo-arbustivas. A IQG apresentou riqueza relativamente baixa $(\mathrm{S}=125)$ comparada a de outras ilhas costeiras do Brasil. Espécies herbáceas (52) e trepadeiras (31) predominaram na IQG. As famílias mais ricas foram Fabaceae (11 espécies), Poaceae (9), Apocynaceae, Asteraceae e Orchidaceae (8 espécies cada). A maioria das espécies ( $\mathrm{S}=112$ ) é autóctone de diferentes fitofisionomias do complexo da Floresta Atlântica do sudeste do Brasil. Muitas espécies associadas a áreas antropicamente alteradas $(\mathrm{S}=26)$ são encontradas na IQG, incluindo a gramínea invasora Melinis minutiflora. Houve ligeira predominância de zoocoria ( $\mathrm{S}=50)$. Nós não identificamos espécies endêmicas para a IQG. Uma espécie (Cattleya intermedia, Orchidaceae) 
encontra-se vulnerável em nível nacional e estadual, e outra (Barrosoa apiculata, Asteraceae) está presumivelmente extinta no estado de São Paulo. A flora vascular da IQG originou-se no complexo da Floresta Atlântica continental, seguindo o padrão de outras ilhas costeiras do sudeste do Brasil. A flora e a vegetação da IQG refletem a combinação da condição insular, tamanho reduzido da ilha, restrição de habitat, topografia acidentada, solos incipientes e o uso pretérito da área com a introdução de várias espécies alóctones. Nós recomendamos o monitoramento permanente da vegetação da IQG e seu manejo, visando garantir a conservação da biota nativa local.

Palavras-chave: Floresta Atlântica; conservação da biodiversidade; ilhas costeiras; síndromes de dispersão; espécies invasoras; áreas protegidas.

\section{Introduction}

Islands have long attracted the attention of scientists and naturalists, and studies of their biota have produced important insights into the interactions of processes and patterns in biogeography (Lomolino 2000). The Equilibrium Theory of Island Biogeography (MacArthur \& Wilson 1967), for example, has strongly influenced studies of ecology and conservation biology through its proposal that the number of species on a given island is the result of both immigration and extinction rates, and that these two opposing forces are closely related to the size and isolation of the island. According to Kreft et al. (2008), the richness of the vascular flora of an island is mainly determined by the island's size, followed by its degree of geographic isolation, current climatic conditions, and its topography and geology. Island biotas are seriously threatened by climate change, habitat loss and, especially, the introduction of invasive alien species (Kreft et al. 2008, Serafini et al. 2010).

Queimada Grande Island (QGI), located off the southern coast of São Paulo State in southeastern Brazil, has stimulated the interests of various researchers in recent decades. The golden lancehead pit viper, Bothrops insularis (Amaral, 1921), is endemic to the island and is critically endangered both nationally (Brasil 2014) and globally (Marques et al. 2004). Although several studies have been carried out on QGI focusing on the biology of this snake (e.g., Wüster et al. 2005, Martins et al. 2008, Marques et al. 2012, Guimarães et al. 2014), information about the island's vegetation cover is still extremely incipient (Martins et al. 2008, Bataus \& Reis 2011). Data on its flora are quite rare in the literature, except for Campos \& Mello-Filho (1966), who presented a study of the flora that referred to approximately twenty (mostly ruderal) species.

Studies of the vegetation on coastal islands in southeastern Brazil have been very scarce (e.g., Barros et al. 1991, Salino et al. 2005, Silva \& Britez 2005, Ferreira et al. 2007, Callado et al. 2009, Bovini et al. 2013, 2014) in spite of their importance for assessing, managing, and conserving biodiversity. Small island plant inventories are even more scarce (Kemenes 2003, Ferreira et al. 2007, Bovini et al. 2014). These studies have frequently indicated that the floras of coastal islands are basically composed of mainland Atlantic Forest complex species (sensu Oliveira-Filho \& Fontes 2000, Scarano 2002), and are related to their recent histories of isolation from the mainland.

We present here and analyze the terrestrial vascular flora of QGI, describe the phytophysiognomies and biodiversity present on the island, and present data useful for managing that area. This contribution was part of a research project conducted jointly by the Instituto de Pesquisas Jardim Botânico do Rio de Janeiro and the Instituto Chico Mendes de Conservação da Biodiversidade (ICMBio) to map and characterize the vegetation cover of QGI and promote the conservation of that island.

\section{Material and Methods}

\section{Study site}

QGI (center point: $24^{\circ} 29^{\prime} 10^{\prime \prime} \mathrm{S}, 46^{\circ} 40^{\prime} 30^{\prime \prime} \mathrm{W}$ ) is located $33.2 \mathrm{~km}$ from the southern coast of São Paulo State in southeastern Brazil (Figure 1). The island has no beaches or plateaus, with very steep slopes and cliffs in its southern

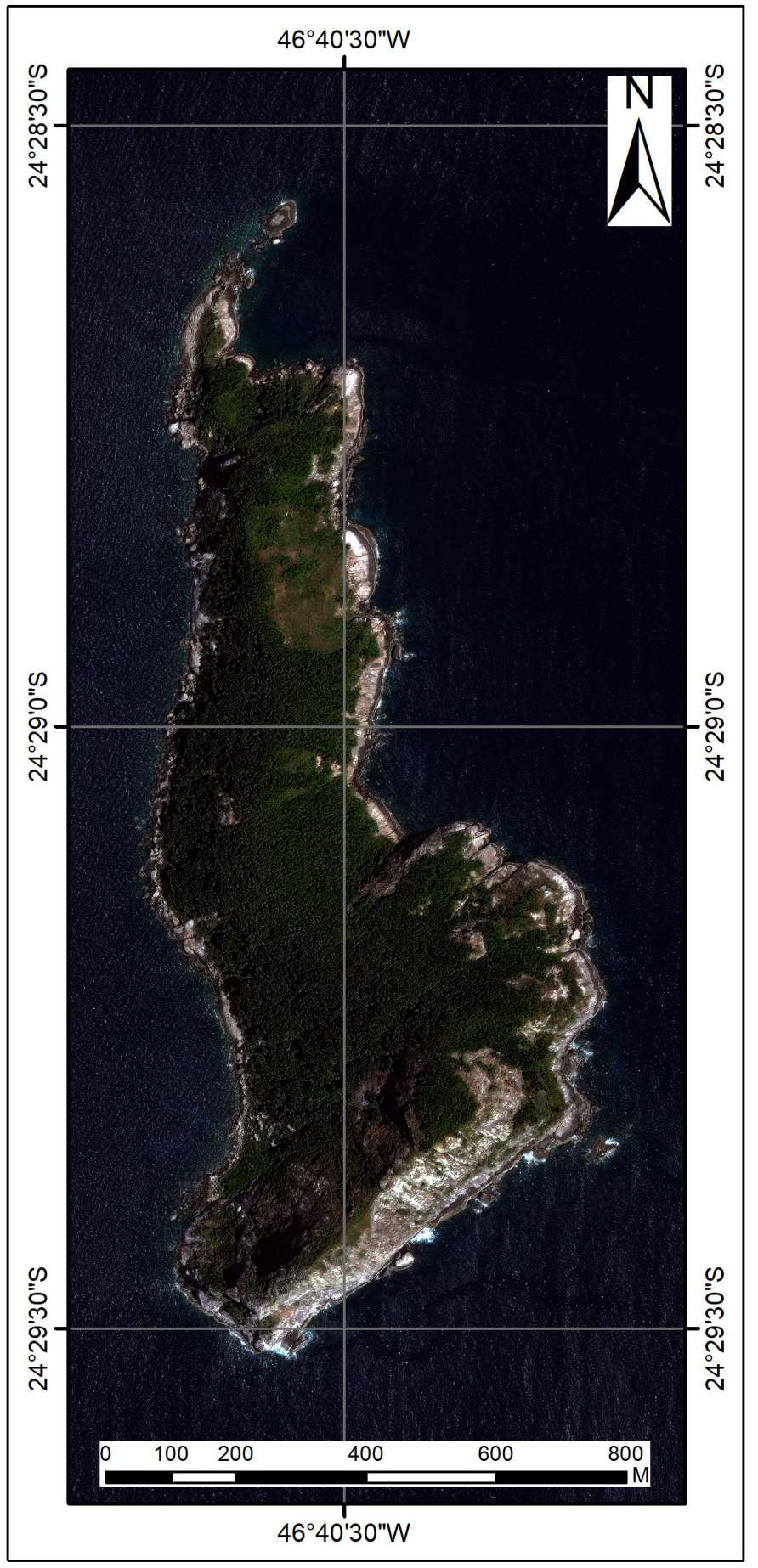

Figure 1. Location of Queimada Grande Island, São Paulo, southeastern Brazil QuickBird image, December 31, 2013, Datum SIRGAS 2000. 
portion (Figure 2) at elevations from 0-210 $\mathrm{m}$ above sea level (Bataus \& Reis 2011). Its projected surface area is 57 ha (B.C. Kurtz et al., unpubl. data) and its soils are shallow, with many boulders and rocky outcrops (pers. obs.). Climate data specific for QGI are extremely scarce. According to Guimarães et al. (2014), the local climate is subtropical with two distinct seasons: rainy and warm (October-March) and dry and cold (AprilSeptember). Average monthly temperatures ranged from $18.3^{\circ} \mathrm{C}$ (August) to $27.2^{\circ} \mathrm{C}$ (March) in 2007-2008, and rainfall from $0.2 \mathrm{~mm}$ (July) to 135.2 $\mathrm{mm}$ (December) (Marques et al. 2012). The general climate for the coast where QGI is located is classified as Af (Tropical, rainforest), following the Köppen's system (Rolim et al. 2007). Itanhaém, for example, the closest city to QGI, has an average annual rainfall of $2030 \mathrm{~mm}$ (Cepagri 2017). The island is relatively distant from the Serra do Mar Range along the mainland coast, however, and should be less affected by orographic rains.

This small coastal island was connected by a land bridge to the mainland during the last glaciation (Wisconsin) maximum about 17,000 years BP, when sea levels were approximately $110 \mathrm{~m}$ lower than today (Tessler \& Goya 2005). The last contact of QGI with the mainland occurred about 11,000 years ago (Marques et al. 2002) when rising sea levels during the Holocene isolated many coastal areas of different sizes and at varying distances from the coastline in southeastern Brazil.

QGI is covered mainly by Dense Ombrophilous Forest (IBGE 2012), with shorter trees than found on the mainland due to local environmental conditions. Additional phytophysiognomies include rock outcrop vegetation (see Meirelles et al. 1999), areas covered by anthropogenic vegetation (characterized by an herbaceous-shrub physiognomy and corresponding to the initial phases of secondary growth as described by IBGE 2012), and a small banana (Musa paradisiaca) plantation (see description below). The latter two formations are the result of past human use of the area.
In 1909, the Brazilian Navy built a lighthouse on QGI, and lighthouse keepers lived there until 1925. The lighthouse has been automated since then and periodically maintained (Bataus \& Reis 2011). QGI was declared part of the Area of Relevant Ecological Interest (AREI) of the Queimada Pequena and Queimada Grande Islands in 1985, a Federal Conservation Area for Sustainable Use managed by ICMBio.

\section{Data collection}

The current floristic list was compiled from collections made on QGI between May/2014 and March/2015 (SISBIO N. 44050-1) and between March/1996 and September/1997, covering all of the seasons of the year. Additionally, samples from the Herbário Virtual da Flora e dos Fungos (INCT 2016) were included, using 'São Paulo' as the search keyword for the state, and 'Ilha Queimada Grande' for the locality. We also included three species that were not collected (found only sterile, or in places of difficult access), but identified in the field. The list was also complemented with information from Wanderley et al. (2001, 2002, 2003, 2005, 2007, $2009,2012,2016)$. The botanical material was identified by the authors and/or specialists. The descriptions of the local phytophysiognomies are based on observations made during field excursions.

\section{Data analysis}

Names and botanical families of all species followed the Flora do Brasil 2020 website (2016) (under construction), which also provided information about life-forms, substrates, and distributions. Species indicated as 'liana/scandent/vine' were treated here simply as climbers (see Morellato \& Leitão-Filho 1996). The conservation statuses of the species at global and national levels followed IUCN (2017) and CNCFlora (2017), respectively. Additionally, we used São Paulo (2016) to confirm the

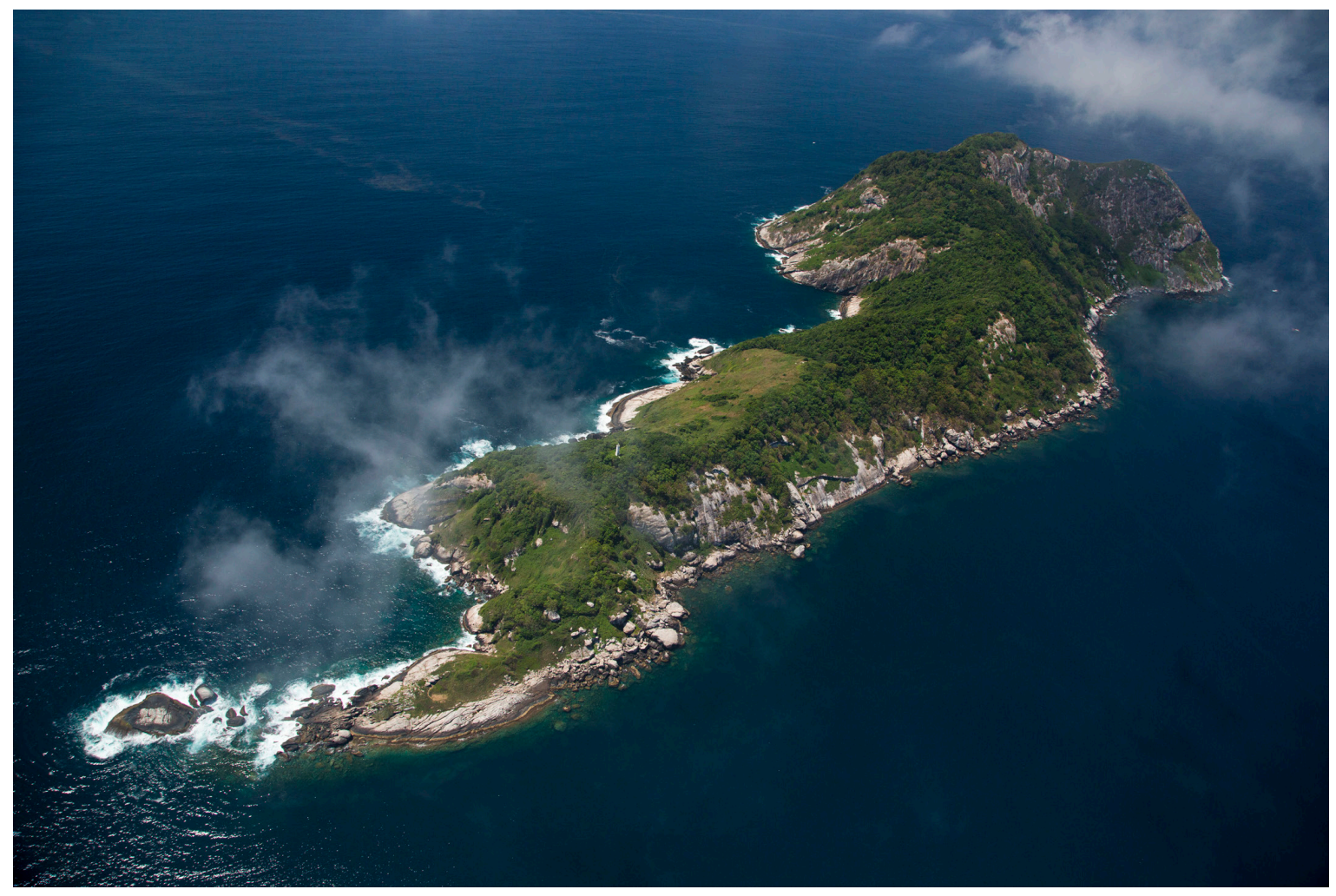

Figure 2. Aerial photograph of Queimada Grande Island, São Paulo, Brazil, showing its rugged topography and its different vegetation physiognomies. Note the area covered by anthropogenic vegetation (in the center of the island near the lighthouse). Photo by J.M. Rosa. 
conservation statuses in São Paulo State. Based on the information about the collection sites found on herbarium labels, species were assigned to one or more of the following habitat classes: forest interior, forest edges (interfaces between forests and open areas), rock outcrop vegetation, anthropogenic vegetation, and banana plantation. By consulting the literature (Ichaso 1980, Pennington et al. 1981, Pennington 1990, Lorenzi 1998, 2000, Barroso et al. 1999, Bovini et al. 2001, Wanderley et al. 2001, 2002, 2003, 2005, 2007, 2009, 2012, 2016, Mansano et al. 2004, Passos \& Oliveira 2004, Rodrigues et al. 2005, Reis 2006, Souza \& Morim 2008, Ferreira 2009, Gomes-Costa \& Alves 2012, Silva-Luz et al. 2012, Ferreira \& Miotto 2013, Soares Neto et al. 2014), specialists, and through examinations of material deposited at the RB Herbarium, the species of angiosperms were classified into four major groups according to the morphological criteria of Pijl (1982): 1) anemochoric, with diaspores adapted to wind dispersal; 2) zoochoric, with diaspores adapted to animal dispersal; 3) hydrochoric, with diaspores adapted to water dispersal; and 4) autochoric, with diaspores displaying no apparent specific adaptation to the above dispersal agents, including barochoric species (gravity dispersal) and those with explosive dispersal. By consulting the same aforementioned sources, we also determined which species are autochthonous to ombrophilous forests and/or pioneer formations (sensu IBGE 2012): beach ridge vegetation (restinga; Lacerda et al. 1993) and rock outcrop vegetation (Meirelles et al. 1999), of the Atlantic Forest complex in southeastern Brazil, and which species are associated with anthropically disturbed areas (ruderal species; sensu Moro et al. 2012). Finally, we identified the invasive alien species in the Atlantic Forest complex that could be competing or displacing populations of native species and hampering vegetation regeneration on QGI, based on Base de Dados Nacional de Espécies Exóticas Invasoras I3N Brasil (Instituto Hórus 2017).

We compared the species richness at QGI with that of other coastal islands in eastern and southeastern Brazil by selecting sites with comprehensive published surveys and physiographies comparable to that of QGI.

\section{Results}

We identified 125 species of vascular plants on QGI, distributed among 115 genera and 57 families, including four pteridophytes and 121 angiosperms (Table 1). The richest families were Fabaceae (11 species), Poaceae (nine species), and Apocynaceae, Asteraceae and Orchidaceae (eight species each). Considering the maximum life-form expression of each species, there was a predominance of herbs $(\mathrm{S}=52 ; 41.6 \%$ of the total $)$ and climbers $(\mathrm{S}=31 ; 24.8 \%)$. Shrubs and subshrubs totaled 25 species (20\%); only 17 species (13.6\%) show arboreal habits. Our results indicated a very low richness of epiphytes on the island, with strictly epiphytic species accounting for only $2.4 \%$ of the local flora $(\mathrm{S}=3$ : Acianthera saundersiana, Epidendrum densiflorum and Peperomia glabella), although this number rises to $8 \%$ if hemiepiphytes (Monstera praetermissa) and facultative epiphytes (i.e., those plants capable of living as rupiculous or terrestrial individuals) with six species (including Rumohra adiantiformis, Aechmea caudata and Cattleya intermedia) are included. QGI showed a relatively low number of species when compared to other coastal islands of eastern and southeastern Brazil (Table 2).

The vast majority of the species ( $\mathrm{S}=112 ; 89.6 \%$ ) are autochthonous from ombrophilous forests and/or pioneer formations (restinga and rock outcrop vegetation) within the Atlantic Forest complex of southeastern Brazil; 13 (10.4\%) species are allochthonous from those formations (e.g., Tetragonia tetragonoides, Crotalaria laeta, Musa paradisiaca and Rivina humilis). Additionally, several species (26) were indicated in the literature (or by specialists) as characteristic of anthropically disturbed areas (e.g., Asclepias curassavica, Bidens pilosa, Ipomoea cairica, Merremia dissecta, Desmodium incanum, Sida rhombifolia, Digitaria insularis and
Paspalum virgatum), with two invasive alien species in the Atlantic Forest complex (Oeceoclades maculata and Melinis minutiflora). Forty species were identified in the local Dense Ombrophilous Forest, and 20 on rock outcrop vegetation. Forty-five species were found in anthropogenic vegetation (including banana plantations) and 37 along forest edges. Some species occurred in more than one habitat. Information concerning the habitats of 7 species was not available (Table 1).

Dense Ombrophilous Forest currently occupies about $50 \%$ of the island area, especially at higher altitude sites and those showing less human interference (Figure 2). Canopy height rarely exceeds $10 \mathrm{~m}$, and species typical of mainland secondary forest formations occur there (such as Guapira opposita, Myrsine guianensis and Gallesia integrifolia). Some forest areas are dominated by the palm tree Syagrus romanzoffiana. The herbaceous layer is rich in Acanthaceae, Orchidaceae, and Araceae. Epiphytes are relatively rare, occurring only sporadically (Lepismium cruciforme and Aechmea caudata).

The rock outcrop vegetation type with an herbaceous or shrub phytophysiognomy occurs on steep slopes or on island peaks, associated with patches of incipient soil accumulation, forming vegetation clumps on the otherwise bare rocky matrix (Figure 3). Closer to the sea, under the influence of salt spray, the rock outcrop vegetation is composed mainly of small shrubs and fleshy herbs, especially Begonia subvillosa, Cereus fernambucensis, and the allochthonous species Tetragonia tetragonoides.

Anthropogenic vegetation, characterized by an herbaceous-shrub physiognomy, represents the early stages of ecological succession in areas originally covered by the Dense Ombrophilous Forest (Figure 2). These areas were formally occupied by rustic houses and small farms (see below), and grasses and other species associated with anthropically disturbed areas predominate there (including Bidens pilosa, Solidago chilensis, Lepidium bonariense, Ipomoea cairica, Eleusine indica and Melinis minutiflora). The anthropogenic vegetation type also includes a small abandoned banana plantation.

Considering only angiosperms, there was a slight predominance of zoochory $(\mathrm{S}=50 ; 41.3 \%)$. Thirty-seven species $(30.6 \%)$ are anemochoric and $33(27.3 \%)$ autochoric, with four hydrochoric species $(3.3 \%)$. These numbers include some species of Poaceae with more than one dispersal syndrome (Table 1). The predominant Dense Ombrophilous Forest showed a preponderance of zoochory $(\mathrm{S}=21 ; 53.8 \%$ of the species surveyed in this vegetation), including species with fleshy fruits (e.g., Eugenia spp., Guapira opposita, Myrsine guianensis, Rudgea minor and Sideroxylon obtusifolium) or arilate seeds (e.g., Trichilia casaretti and Cupania oblongifolia) adapted to endozoochory. Zoochoric species, on the other hand, occurring in the anthropogenic vegetation and along forest edges showed different dispersal strategies, including structures that facilitate the adhesion of their diaspores to animals (or clothes) (e.g., Cyathula prostrata, Bidens spp., Desmodium incanum and Sida spp.).

This study did not detect any plant species endemic to QGI. Most of the species found on QGI have not yet been evaluated in terms of their conservation statuses at a national level, and only one species (Cattleya intermedia) is considered vulnerable (VU) at both national and regional levels. The only record of this orchid on the island is from 1922 (A. Gehrt $\mathrm{s} / \mathrm{n}, \mathrm{SP}$ 8146), and it may be presumed to be extinct on QGI, as it has not been found during recent expeditions. Additionally, Barrosoa apiculata is considered extinct (EX) in São Paulo State according to the official list of threatened species of São Paulo. The only known specimen of this species was collected on the island in 1920 (A. Gehrt s/n, SP 4535) and our collecting efforts failed to find any additional individuals. Although Trichilia casaretti has been classified as vulnerable (VU) at a global level, its status needs updating according to IUCN (2017). 
Table 1. List of the vascular flora of Queimada Grande Island, São Paulo, Brazil. Species marked with an '*' are indicated in the literature as characteristic of anthropically disturbed areas, and species marked with a ' $\dagger$ ' are invasive in the Atlantic Forest complex in southeastern Brazil. Substrate: Epi (epiphytic); Hem (hemiepiphytes); Rup (rupiculous); Ter (terrestrial). Origin: Auto (autochthonous); Allo (allochthonous) from the Atlantic Forest complex in southeastern Brazil. Dispersal syndrome: Anemo (anemochoric); Auto (autochoric); Hydro (hydrochoric); Zoo (zoochoric). Conservation statuses at global [square brackets], national (no brackets), and regional \{curly brackets\} levels: NE (Not Evaluated); LC (Least Concern); VU (Vulnerable); EX (Presumably Extinct). Herbaria: ESA (Escola Superior de Agricultura Luiz de Queiroz); NY (The New York Botanical Garden); RB (Instituto de Pesquisas Jardim Botânico do Rio de Janeiro); SP (Herbário do Estado Maria Eneyda P. Kaufmann Fidalgo); SPF (Universidade de São Paulo); UEC (Universidade Estadual de Campinas).

\begin{tabular}{|c|c|c|c|c|c|c|c|c|}
\hline Family & Species & Life-form & Substrate & Origin & Habitat & Syndrome & Status & Voucher \\
\hline \multicolumn{9}{|l|}{ PTERIDOPHYTES } \\
\hline Aspleniaceae & $\begin{array}{c}\text { Asplenium claussenii } \\
\text { Hieron. }\end{array}$ & Herb & Rup, Ter & Auto & Forest & - & NE & $\begin{array}{l}\text { V.C. Souza 11067: } \\
\text { RB } 629051\end{array}$ \\
\hline Blechnaceae & $\begin{array}{c}\text { Blechnum polypodioides } \\
\text { Raddi }\end{array}$ & Herb & Ter & Auto & No information & - & NE & $\begin{array}{l}\text { A. Gehrt } 4545 \text { : } \\
\text { NY } 814169\end{array}$ \\
\hline Dryopteridaceae & $\begin{array}{l}\text { Rumohra adiantiformis } \\
\text { (G. Forst.) Ching }\end{array}$ & Herb & $\begin{array}{l}\text { Epi, Rup, } \\
\text { Ter }\end{array}$ & Auto & $\begin{array}{l}\text { Rock outcrop } \\
\text { vegetation }\end{array}$ & - & NE & $\begin{array}{l}\text { V.C. Souza 11037: } \\
\text { RB } 611809\end{array}$ \\
\hline Polypodiaceae & $\begin{array}{l}\text { Serpocaulon triseriale } \\
\text { (Sw.) A.R. Sm. }\end{array}$ & Herb & Rup, Ter & Auto & Forest edge & - & NE & $\begin{array}{l}\text { V.C. Souza 11054: } \\
\text { RB } 627263\end{array}$ \\
\hline \multicolumn{9}{|l|}{ ANGIOSPERMS } \\
\hline \multirow[t]{4}{*}{ Acanthaceae } & $\begin{array}{c}\text { Dicliptera mucronifolia } \\
\text { Nees }\end{array}$ & Subshrub & Ter & Auto & Forest & Auto & NE & $\begin{array}{l}\text { G.O. Joaquim Jr. } \\
\text { 16: RB } 627046\end{array}$ \\
\hline & $\begin{array}{c}\text { Justicia kleinii Wassh. \& } \\
\text { L.B. Sm. }\end{array}$ & Herb & Ter & Auto & Forest & Auto & $\mathrm{LC}$ & $\begin{array}{l}\text { A.M. Magalhães } \\
65: \text { RB } 636590\end{array}$ \\
\hline & $\begin{array}{c}\text { Pseuderanthemum } \\
\text { heterophyllum (Nees) } \\
\text { Radlk. }\end{array}$ & Herb & Ter & Auto & Forest & Auto & NE & $\begin{array}{l}\text { G.O. Joaquim Jr. } \\
\text { 39: ESA } 33144\end{array}$ \\
\hline & $\begin{array}{l}\text { Ruellia brevifolia (Pohl) } \\
\text { C. Ezcurra }\end{array}$ & Subshrub & Ter & Auto & Forest edge & Auto & NE & $\begin{array}{l}\text { G.O. Joaquim Jr. } \\
\text { 123: RB } 627016\end{array}$ \\
\hline \multirow[t]{2}{*}{ Aizoaceae } & $\begin{array}{l}\text { Sesuvium portulacastrum } \\
\text { (L.) L. }\end{array}$ & Herb & Rup, Ter & Auto & $\begin{array}{l}\text { Rock outcrop } \\
\text { vegetation }\end{array}$ & Auto & $\mathrm{NE}$ & $\begin{array}{l}\text { V.C. Souza 11045: } \\
\text { ESA } 27428\end{array}$ \\
\hline & $\begin{array}{l}\text { Tetragonia tetragonoides } \\
\text { (Pall.) Kuntze }\end{array}$ & Herb & Ter & Allo & $\begin{array}{l}\text { Rock outcrop } \\
\text { vegetation }\end{array}$ & Hydro & $\mathrm{NE}$ & $\begin{array}{l}\text { V.C. Souza 11032: } \\
\text { ESA } 31994\end{array}$ \\
\hline \multirow[t]{3}{*}{ Amaranthaceae } & $\begin{array}{l}\text { Alternanthera brasiliana } \\
\text { (L.) Kuntze }\end{array}$ & Subshrub & Ter & Auto & $\begin{array}{l}\text { Anthropogenic } \\
\text { vegetation }\end{array}$ & Auto & NE & $\begin{array}{l}\text { A. Gehrt s/n: } \\
\text { SP } 4565\end{array}$ \\
\hline & $\begin{array}{c}\text { Cyathula prostrata } \\
\text { Blume* }\end{array}$ & Subshrub & Ter & Auto & $\begin{array}{l}\text { Anthropogenic } \\
\text { vegetation }\end{array}$ & Zoo & $\mathrm{NE}$ & $\begin{array}{l}\text { A.M. Magalhães } \\
\text { 38: RB } 636575\end{array}$ \\
\hline & $\begin{array}{l}\text { Dysphania ambrosioides } \\
\text { (L.) Mosyakin \& } \\
\text { Clemants }\end{array}$ & Subshrub & Ter & Allo & $\begin{array}{l}\text { Anthropogenic } \\
\text { vegetation }\end{array}$ & Zoo & NE & $\begin{array}{l}\text { A. Gehrt s/n: } \\
\text { SP } 4564\end{array}$ \\
\hline Amaryllidaceae & $\begin{array}{c}\text { Hippeastrum reticulatum } \\
\text { Herb. }\end{array}$ & Herb & Ter & Auto & Forest & Auto & NE & $\begin{array}{l}\text { A.M. Magalhães } \\
\text { 66: RB } 636591\end{array}$ \\
\hline \multirow[t]{8}{*}{ Apocynaceae } & Asclepias curassavica L.* & Herb & Ter & Auto & $\begin{array}{l}\text { Anthropogenic } \\
\text { vegetation }\end{array}$ & Anemo & NE & $\begin{array}{l}\text { A.M. Magalhães } \\
\text { 51: RB } 636582\end{array}$ \\
\hline & $\begin{array}{l}\text { Aspidosperma australe } \\
\text { Müll. Arg. }\end{array}$ & Tree & Ter & Auto & Forest & Anemo & $\mathrm{LC}$ & $\begin{array}{l}\text { A.M. Magalhães } \\
\text { 96: RB } 636611\end{array}$ \\
\hline & $\begin{array}{l}\text { Forsteronia thyrsoidea } \\
\text { (Vell.) Müll. Arg. }\end{array}$ & Climber & Ter & Auto & Forest edge & Anemo & $\mathrm{NE}$ & $\begin{array}{l}\text { A. Gehrt s/n: } \\
\text { RB } 119773\end{array}$ \\
\hline & $\begin{array}{l}\text { Gonolobus rostratus } \\
\text { (Vahl) R. Br. ex Shult. }\end{array}$ & Climber & Ter & Auto & $\begin{array}{l}\text { Forest edge, } \\
\text { Anthropogenic } \\
\text { vegetation }\end{array}$ & Anemo & NE & $\begin{array}{l}\text { A.M. Magalhães } \\
\text { 99: RB } 636613\end{array}$ \\
\hline & $\begin{array}{l}\text { Jobinia connivens (Hook. } \\
\qquad \text { A Arn.) Malme }\end{array}$ & Climber & Ter & Auto & Forest edge & Anemo & NE & $\begin{array}{l}\text { G.O. Joaquim Jr. } \\
\text { 127: ESA } 34562\end{array}$ \\
\hline & $\begin{array}{l}\text { Marsdenia macrophylla } \\
\text { (Humb. \& Bonpl. ex } \\
\text { Schult.) E. Fourn. }\end{array}$ & Climber & Ter & Auto & Forest edge & Anemo & NE & $\begin{array}{l}\text { A. Gehrt s/n: } \\
\text { SP } 28663\end{array}$ \\
\hline & $\begin{array}{c}\text { Orthosia urceolata E. } \\
\text { Fourn. }\end{array}$ & Climber & Ter & Auto & Forest edge & Anemo & NE & $\begin{array}{l}\text { G.O. Joaquim Jr. } \\
\text { 115: ESA } 34577\end{array}$ \\
\hline & $\begin{array}{l}\text { Temnadenia odorifera } \\
\text { (Vell.) J.F. Morales }\end{array}$ & Climber & Ter & Auto & $\begin{array}{l}\text { Anthropogenic } \\
\text { vegetation }\end{array}$ & Anemo & NE & $\begin{array}{l}\text { A.M. Magalhães } \\
\text { 74: RB } 637080\end{array}$ \\
\hline \multirow[t]{3}{*}{ Araceae } & $\begin{array}{l}\text { Anthurium parasiticum } \\
\text { (Vell.) Stellfeld }\end{array}$ & Herb & Ter & Auto & Forest & Zoo & $\mathrm{LC}$ & $\begin{array}{l}\text { V.C. Souza 11091: } \\
\text { RB } 427676\end{array}$ \\
\hline & $\begin{array}{l}\text { Monstera praetermissa } \\
\text { E.G. Gonç. \& Temponi }\end{array}$ & Herb & Hem & Auto & Forest & Zoo & NE & $\begin{array}{l}\text { V.C. Souza 11041: } \\
\text { ESA } 27427\end{array}$ \\
\hline & Xanthosoma sp. & Herb & & Auto & $\begin{array}{l}\text { Rock outcrop } \\
\text { vegetation, } \\
\text { Anthropogenic } \\
\text { vegetation }\end{array}$ & Zoo & & Not collected \\
\hline
\end{tabular}


Kurtz B.C. et al.

Table 1. Continued...

\begin{tabular}{|c|c|c|c|c|c|c|c|c|}
\hline Family & Species & Life-form & Substrate & Origin & Habitat & Syndrome & Status & Voucher \\
\hline Arecaceae & $\begin{array}{l}\text { Syagrus romanzoffiana } \\
\text { (Cham.) Glassman }\end{array}$ & Palm Tree & Ter & Auto & Forest & Zoo & $\mathrm{LC}$ & Not collected \\
\hline \multirow[t]{8}{*}{ Asteraceae } & $\begin{array}{c}\text { Austroeupatorium } \\
\text { inulaefolium (Kunth) } \\
\text { R.M. King \& H. Rob. }\end{array}$ & $\begin{array}{l}\text { Shrub, } \\
\text { Subshrub }\end{array}$ & Rup, Ter & Auto & $\begin{array}{l}\text { Forest edge, } \\
\text { Anthropogenic } \\
\text { vegetation }\end{array}$ & Anemo & $\mathrm{NE}$ & $\begin{array}{c}\text { A.M. Magalhães 21: } \\
\text { RB } 637078\end{array}$ \\
\hline & $\begin{array}{c}\text { Barrosoa apiculata } \\
\text { (Gardner) R.M. King \& } \\
\text { H. Rob. }\end{array}$ & Herb & Ter & Auto & $\begin{array}{l}\text { Rock outcrop } \\
\text { vegetation }\end{array}$ & Anemo & $\begin{array}{c}\mathrm{LC} \\
\{\mathrm{EX}\}\end{array}$ & $\begin{array}{l}\text { A. Gehrt s/n: } \\
\text { SP } 4535\end{array}$ \\
\hline & Bidens pilosa L.* & Herb & Ter & Allo & $\begin{array}{c}\text { Anthropogenic } \\
\text { vegetation }\end{array}$ & Zoo & $\mathrm{NE}$ & $\begin{array}{l}\text { V.C. Souza 11015: } \\
\quad \text { ESA } 26128\end{array}$ \\
\hline & $\begin{array}{l}\text { Bidens segetum Mart. ex } \\
\text { Colla }\end{array}$ & $\begin{array}{l}\text { Climber, } \\
\text { Subshrub }\end{array}$ & Ter & Auto & $\begin{array}{c}\text { Anthropogenic } \\
\text { vegetation }\end{array}$ & Zoo & $\mathrm{NE}$ & $\begin{array}{l}\text { A.M. Magalhães } \\
\text { 39: RB } 636576\end{array}$ \\
\hline & $\begin{array}{c}\text { Chromolaena odorata } \\
\text { (L.) R.M. King \& H. } \\
\text { Rob.* }\end{array}$ & Shrub & Ter & Auto & $\begin{array}{l}\text { Forest edge, } \\
\text { Anthropogenic } \\
\text { vegetation }\end{array}$ & Anemo & $\mathrm{NE}$ & $\begin{array}{l}\text { A.M. Magalhães } \\
\text { 24: RB } 636569\end{array}$ \\
\hline & $\begin{array}{l}\text { Cyrtocymura scorpioides } \\
\text { (Lam.) H. Rob. }\end{array}$ & Subshrub & Ter & Auto & $\begin{array}{l}\text { Anthropogenic } \\
\text { vegetation }\end{array}$ & Anemo & $\mathrm{NE}$ & $\begin{array}{l}\text { A.M. Magalhães } \\
\text { 59: RB } 636585\end{array}$ \\
\hline & $\begin{array}{c}\text { Mikania micrantha } \\
\text { Kunth* }\end{array}$ & Climber & Ter & Auto & $\begin{array}{l}\text { Anthropogenic } \\
\text { vegetation }\end{array}$ & Anemo & $\mathrm{NE}$ & $\begin{array}{l}\text { A.M. Magalhães } \\
\text { 44: RB } 636579\end{array}$ \\
\hline & $\begin{array}{c}\text { Solidago chilensis } \\
\text { Meyen* }\end{array}$ & Subshrub & Ter & Auto & $\begin{array}{l}\text { Anthropogenic } \\
\text { vegetation }\end{array}$ & Anemo & $\mathrm{NE}$ & $\begin{array}{c}\text { V.C. Souza 11069: } \\
\text { ESA } 26144\end{array}$ \\
\hline Basellaceae & $\begin{array}{l}\text { Anredera cordifolia } \\
\text { (Ten.) Steenis }\end{array}$ & Climber & Ter & Auto & Forest edge & Auto & $\mathrm{NE}$ & $\begin{array}{l}\text { V.C. Souza 11021: } \\
\text { ESA } 26119\end{array}$ \\
\hline \multirow[t]{2}{*}{ Begoniaceae } & $\begin{array}{c}\text { Begonia fernandocostae } \\
\text { Irmsch. }\end{array}$ & Subshrub & Ter & Auto & No information & Anemo & $\mathrm{NE}$ & $\begin{array}{l}\text { A. Amaral \& J. } \\
\text { Domingues 26a: } \\
\quad \text { SP } 3946\end{array}$ \\
\hline & $\begin{array}{c}\text { Begonia subvillosa } \\
\text { Klotzsch }\end{array}$ & Herb & Rup, Ter & Auto & $\begin{array}{c}\text { Forest, Rock } \\
\text { outcrop vegetation }\end{array}$ & Anemo & $\mathrm{NE}$ & $\begin{array}{l}\text { V.C. Souza 11011: } \\
\quad \text { ESA } 26125\end{array}$ \\
\hline Bignoniaceae & $\begin{array}{l}\text { Dolichandra quadrivalvis } \\
\text { (Jacq.) L.G. Lohmann }\end{array}$ & Climber & Ter & Auto & Forest & Anemo & $\mathrm{NE}$ & $\begin{array}{l}\text { A. Gehrt s/n: } \\
\text { SP } 4556\end{array}$ \\
\hline \multirow[t]{2}{*}{ Boraginaceae } & $\begin{array}{c}\text { Tournefortia } \\
\text { membranacea (Gardner) } \\
\text { DC. }\end{array}$ & $\begin{array}{l}\text { Shrub, } \\
\text { Climber, } \\
\text { Subshrub }\end{array}$ & Ter & Auto & Forest edge & Zoo & $\mathrm{NE}$ & $\begin{array}{l}\text { V.C. Souza 11090: } \\
\text { ESA } 26155\end{array}$ \\
\hline & $\begin{array}{c}\text { Varronia curassavica } \\
\text { Jacq. }\end{array}$ & Shrub & Ter & Auto & $\begin{array}{l}\text { Forest edge, } \\
\text { Anthropogenic } \\
\text { vegetation }\end{array}$ & Zoo & $\mathrm{NE}$ & $\begin{array}{l}\text { D.F. Bertani s/n: } \\
\text { RB } 552595\end{array}$ \\
\hline Brassicaceae & Lepidium bonariense L.* & Herb & Ter & Auto & $\begin{array}{l}\text { Anthropogenic } \\
\text { vegetation }\end{array}$ & Zoo & $\mathrm{NE}$ & $\begin{array}{l}\text { G.O. Joaquim Jr. } \\
\text { 14: ESA } 33163\end{array}$ \\
\hline Bromeliaceae & Aechmea caudata Lindm. & Herb & $\begin{array}{l}\text { Epi, Rup, } \\
\text { Ter }\end{array}$ & Auto & Forest & Zoo & $\mathrm{LC}$ & $\begin{array}{l}\text { V.C. Souza 11022: } \\
\text { RB } 471967\end{array}$ \\
\hline \multirow[t]{4}{*}{ Cactaceae } & $\begin{array}{c}\text { Cereus fernambucensis } \\
\text { Lem. }\end{array}$ & $\begin{array}{l}\text { Shrub, } \\
\text { Subshrub }\end{array}$ & Rup, Ter & Auto & $\begin{array}{l}\text { Rock outcrop } \\
\text { vegetation }\end{array}$ & Zoo & $\mathrm{NE}[\mathrm{LC}]$ & $\begin{array}{l}\text { A. Gehrt s/n: } \\
\text { SP } 4574\end{array}$ \\
\hline & $\begin{array}{c}\text { Coleocephalocereus } \\
\text { fluminensis (Miq.) } \\
\text { Backeb. }\end{array}$ & Shrub & Rup & Auto & $\begin{array}{l}\text { Rock outcrop } \\
\text { vegetation }\end{array}$ & Zoo & $\mathrm{NE}[\mathrm{LC}]$ & $\begin{array}{l}\text { A.M. Magalhães } \\
\text { 94: RB } 636609\end{array}$ \\
\hline & $\begin{array}{c}\text { Lepismium cruciforme } \\
\text { (Vell.) Miq. }\end{array}$ & Herb & Epi, Rup & Auto & Forest & Zoo & $\mathrm{LC}[\mathrm{LC}]$ & $\begin{array}{c}\text { V.C. Souza 11060: } \\
\text { ESA } 26136\end{array}$ \\
\hline & Pereskia aculeata Mill. & Climber & Rup, Ter & Auto & Forest, Forest edge & Zoo & $\mathrm{LC}[\mathrm{LC}]$ & $\begin{array}{l}\text { A.M. Magalhães } \\
\text { 76: RB } 636598\end{array}$ \\
\hline Campanulaceae & $\begin{array}{l}\text { Hippobroma longiflora } \\
\text { (L.) G. Don* }\end{array}$ & Herb, Shrub & Ter & Auto & $\begin{array}{l}\text { Anthropogenic } \\
\text { vegetation }\end{array}$ & Auto & $\mathrm{NE}$ & $\begin{array}{l}\text { A.M. Magalhães } \\
\text { 62: RB } 636588\end{array}$ \\
\hline Cannabaceae & $\begin{array}{c}\text { Trema micrantha (L.) } \\
\text { Blume }\end{array}$ & Shrub, Tree & Ter & Auto & Forest & Zoo & $\mathrm{NE}$ & $\begin{array}{c}\text { V.C. Souza 11058: } \\
\text { ESA } 26153\end{array}$ \\
\hline Cannaceae & Canna indica $\mathrm{L}$. & Herb & Ter & Auto & $\begin{array}{l}\text { Forest edge, } \\
\text { Anthropogenic } \\
\text { vegetation }\end{array}$ & Auto & $\mathrm{NE}$ & $\begin{array}{l}\text { A.M. Magalhães } \\
\text { 63: RB } 636589\end{array}$ \\
\hline Capparaceae & $\begin{array}{c}\text { Cynophalla flexuosa (L.) } \\
\text { J. Presl }\end{array}$ & Shrub & Ter & Auto & Forest & Zoo & $\mathrm{NE}$ & $\begin{array}{c}\text { V.C. Souza 11068: } \\
\text { ESA } 26143\end{array}$ \\
\hline Celastraceae & Hippocratea volubilis L. & Climber & Ter & Auto & Forest edge & Anemo & $\mathrm{NE}$ & $\begin{array}{c}\text { V.C. Souza 11030: } \\
\text { ESA } 27433\end{array}$ \\
\hline Cleomaceae & $\begin{array}{c}\text { Cleome rosea Vahl ex } \\
\text { DC. }\end{array}$ & $\begin{array}{c}\text { Herb, } \\
\text { Subshrub }\end{array}$ & Rup, Ter & Auto & $\begin{array}{l}\text { Rock outcrop } \\
\text { vegetation }\end{array}$ & Zoo & $\mathrm{NE}$ & $\begin{array}{c}\text { V.C. Souza 11012: } \\
\text { ESA } 26126\end{array}$ \\
\hline
\end{tabular}


Table 1. Continued...

\begin{tabular}{|c|c|c|c|c|c|c|c|c|}
\hline Family & Species & Life-form & Substrate & Origin & Habitat & Syndrome & Status & Voucher \\
\hline & $\begin{array}{c}\text { Hemiscola aculeata (L.) } \\
\text { Raf.* }\end{array}$ & Herb & Rup, Ter & Auto & Forest edge & Zoo & NE & $\begin{array}{l}\text { A. Gehrt s/n: } \\
\text { SPF } 100854\end{array}$ \\
\hline \multirow[t]{3}{*}{ Commelinaceae } & Commelina erecta $\mathrm{L} . *$ & Herb & Rup, Ter & Auto & $\begin{array}{l}\text { Forest edge, Rock } \\
\text { outcrop vegetation }\end{array}$ & Auto & $\mathrm{NE}[\mathrm{LC}]$ & $\begin{array}{l}\text { A.M. Magalhães } \\
\text { 95: RB } 636610\end{array}$ \\
\hline & $\begin{array}{c}\text { Gibasis geniculata (Jacq.) } \\
\text { Rohweder }\end{array}$ & Herb & Rup, Ter & Auto & Forest edge & Auto & $\mathrm{NE}$ & $\begin{array}{l}\text { V.C. Souza } 11092 \text { : } \\
\text { ESA } 26157\end{array}$ \\
\hline & $\begin{array}{c}\text { Tradescantia fluminensis } \\
\text { Vell. }\end{array}$ & Herb & $\begin{array}{l}\text { Epi, Rup, } \\
\quad \text { Ter }\end{array}$ & Auto & Forest & Auto & $\mathrm{NE}$ & $\begin{array}{c}\text { V.C. Souza 11093: } \\
\text { ESA } 26158\end{array}$ \\
\hline \multirow[t]{5}{*}{ Convolvulaceae } & $\begin{array}{c}\text { Ipomoea cairica }(\mathrm{L} .) \\
\text { Sweet* }\end{array}$ & Climber & Ter & Auto & $\begin{array}{c}\text { Anthropogenic } \\
\text { vegetation }\end{array}$ & Anemo & $\mathrm{NE}$ & $\begin{array}{c}\text { G.O. Joaquim Jr. 113: } \\
\text { ESA } 34579\end{array}$ \\
\hline & $\begin{array}{c}\text { Ipomoea tiliacea (Willd.) } \\
\text { Choisy* }\end{array}$ & Climber & Ter & Auto & Forest edge & Anemo & $\mathrm{NE}$ & $\begin{array}{c}\text { V.C. Souza 11066: } \\
\text { ESA } 70940\end{array}$ \\
\hline & Ipomoea triloba L.* & Climber & Ter & Allo & $\begin{array}{c}\text { Anthropogenic } \\
\text { vegetation }\end{array}$ & Auto & $\mathrm{NE}$ & $\begin{array}{l}\text { G.O. Joaquim Jr. } \\
\text { 129: ESA } 87102\end{array}$ \\
\hline & $\begin{array}{c}\text { Jacquemontia ferruginea } \\
\text { Choisy }\end{array}$ & Climber & Ter & Auto & $\begin{array}{l}\text { Forest edge, } \\
\text { Anthropogenic } \\
\text { vegetation }\end{array}$ & Auto & $\mathrm{NE}$ & $\begin{array}{l}\text { A.M. Magalhães } \\
\text { 68: RB } 636593\end{array}$ \\
\hline & $\begin{array}{c}\text { Merremia dissecta (Jacq.) } \\
\text { Hallier f.* }\end{array}$ & Climber & Ter & Auto & $\begin{array}{l}\text { Forest edge, } \\
\text { Anthropogenic } \\
\text { vegetation }\end{array}$ & Auto & $\mathrm{NE}$ & $\begin{array}{l}\text { A.M. Magalhães 85: } \\
\text { RB } 636601\end{array}$ \\
\hline Cucurbitaceae & Melothria pendula L.* & Climber & Ter & Auto & Forest edge & Zoo & $\mathrm{NE}$ & $\begin{array}{c}\text { A.M. Magalhães } \\
\text { 64: RB } 637079\end{array}$ \\
\hline Cyperaceae & Cyperus ligularis L. & Herb & Rup, Ter & Auto & $\begin{array}{c}\text { Anthropogenic } \\
\text { vegetation }\end{array}$ & Zoo & $\mathrm{NE}$ & $\begin{array}{c}\text { A.M. Magalhães 78: } \\
\text { RB } 636599\end{array}$ \\
\hline Dioscoreaceae & $\begin{array}{c}\text { Dioscorea fodinarum } \\
\text { Kunth }\end{array}$ & Climber & Ter & Auto & Forest edge & Anemo & $\mathrm{NE}$ & $\begin{array}{c}\text { V.C. Souza 11086: } \\
\text { ESA } 27114\end{array}$ \\
\hline Ebenaceae & $\begin{array}{l}\text { Diospyros inconstans } \\
\text { Jacq. }\end{array}$ & Tree & Ter & Auto & Forest, Forest edge & Zoo & $\mathrm{LC}$ & $\begin{array}{l}\text { V.C. Souza 11010: } \\
\text { ESA } 26134\end{array}$ \\
\hline Erythroxylaceae & $\begin{array}{c}\text { Erythroxylum } \\
\text { cuspidifolium Mart. }\end{array}$ & Shrub, Tree & Ter & Auto & Forest & Zoo & $\mathrm{NE}$ & $\begin{array}{l}\text { V.C. Souza 11078: } \\
\text { ESA } 27107\end{array}$ \\
\hline \multirow[t]{3}{*}{ Euphorbiaceae } & $\begin{array}{c}\text { Algernonia riedelii (Müll. } \\
\text { Arg.) G.L. Webster }\end{array}$ & Tree & Ter & Auto & Forest & Auto & $\mathrm{NE}$ & $\begin{array}{l}\text { V.C. Souza 11087: } \\
\text { ESA } 27115\end{array}$ \\
\hline & Euphorbia insulana Vell. & Herb & Ter & Auto & $\begin{array}{c}\text { Anthropogenic } \\
\text { vegetation }\end{array}$ & Auto & $\mathrm{NE}$ & $\begin{array}{c}\text { V.C. Souza 11061: } \\
\text { ESA } 26137\end{array}$ \\
\hline & Tragia volubilis L. & Climber & Epi, Ter & Auto & Forest edge & Auto & $\mathrm{NE}$ & $\begin{array}{c}\text { A. Amaral \& D. } \\
\text { Lemos s/n: SP } 3887\end{array}$ \\
\hline \multirow[t]{11}{*}{ Fabaceae } & $\begin{array}{c}\text { Canavalia rosea (Sw.) } \\
\text { DC. }\end{array}$ & $\begin{array}{l}\text { Herb, } \\
\text { Climber }\end{array}$ & Ter & Auto & $\begin{array}{l}\text { Rock outcrop } \\
\text { vegetation }\end{array}$ & Hydro & $\mathrm{NE}$ & $\begin{array}{c}\text { V.C. Souza 11031: } \\
\text { ESA } 27434\end{array}$ \\
\hline & $\begin{array}{l}\text { Centrosema virginianum } \\
\text { (L.) Benth. }\end{array}$ & Climber & Ter & Auto & $\begin{array}{c}\text { Anthropogenic } \\
\text { vegetation }\end{array}$ & Auto & $\mathrm{NE}$ & $\begin{array}{l}\text { G.O. Joaquim Jr. } \\
\text { 122: RB } 587987\end{array}$ \\
\hline & $\begin{array}{c}\text { Chaetocalyx brasiliensis } \\
\text { (Vogel) Benth. }\end{array}$ & Climber & Ter & Auto & $\begin{array}{l}\text { Rock outcrop } \\
\text { vegetation }\end{array}$ & Auto & $\mathrm{NE}$ & $\begin{array}{l}\text { A. Gehrt s/n: } \\
\text { UEC } 84324\end{array}$ \\
\hline & $\begin{array}{c}\text { Condylostylis candida } \\
\text { (Vell.) A. Delgado }\end{array}$ & Climber & Ter & Auto & Forest edge & Auto & $\mathrm{NE}$ & $\begin{array}{l}\text { V.C. Souza 11096: } \\
\text { ESA } 26161\end{array}$ \\
\hline & $\begin{array}{c}\text { Crotalaria laeta Mart. ex } \\
\text { Benth. }\end{array}$ & Subshrub & Ter & Allo & $\begin{array}{l}\text { Anthropogenic } \\
\text { vegetation }\end{array}$ & Auto & $\mathrm{NE}$ & $\begin{array}{l}\text { A.M. Magalhães } \\
\text { 75: RB } 636597\end{array}$ \\
\hline & $\begin{array}{l}\text { Crotalaria vitellina Ker } \\
\text { Gawl. }\end{array}$ & $\begin{array}{l}\text { Shrub, } \\
\text { Subshrub }\end{array}$ & Ter & Auto & $\begin{array}{l}\text { Forest edge, Rock } \\
\text { outcrop vegetation }\end{array}$ & Auto & $\mathrm{NE}$ & $\begin{array}{l}\text { F.T. Farah 30: } \\
\text { RB } 593080\end{array}$ \\
\hline & $\begin{array}{l}\text { Desmodium incanum } \\
\text { (Sw.) DC.* }\end{array}$ & Subshrub & Ter & Allo & $\begin{array}{c}\text { Forest edge, } \\
\text { Anthropogenic } \\
\text { vegetation }\end{array}$ & Zoo & $\mathrm{NE}$ & $\begin{array}{l}\text { A.M. Magalhães } \\
\text { 46: RB } 636580\end{array}$ \\
\hline & $\begin{array}{c}\text { Senegalia tenuifolia (L.) } \\
\text { Britton \& Rose }\end{array}$ & $\begin{array}{l}\text { Shrub, } \\
\text { Climber }\end{array}$ & Ter & Auto & $\begin{array}{c}\text { Forest, Forest edge, } \\
\text { Anthropogenic } \\
\text { vegetation }\end{array}$ & Auto & $\mathrm{NE}$ & $\begin{array}{c}\text { A.M. Magalhães 69: } \\
\text { RB } 636594\end{array}$ \\
\hline & $\begin{array}{l}\text { Senna neglecta (Vogel) } \\
\text { H.S. Irwin \& Barneby* }\end{array}$ & Shrub & Rup, Ter & Auto & $\begin{array}{l}\text { Anthropogenic } \\
\text { vegetation }\end{array}$ & Auto & $\mathrm{NE}$ & $\begin{array}{l}\text { V.C. Souza 11085: } \\
\quad \text { RB 591824 }\end{array}$ \\
\hline & $\begin{array}{l}\text { Sigmoidotropis speciosa } \\
\text { (Kunth) A. Delgado }\end{array}$ & Climber & Ter & Auto & $\begin{array}{l}\text { Rock outcrop } \\
\text { vegetation }\end{array}$ & Auto & $\mathrm{NE}$ & $\begin{array}{l}\text { A. Gehrt s/n: } \\
\text { SP } 4553\end{array}$ \\
\hline & $\begin{array}{c}\text { Zollernia ilicifolia } \\
\text { (Brongn.) Vogel }\end{array}$ & Shrub, Tree & Ter & Auto & Forest & Zoo & $\mathrm{NE}$ & $\begin{array}{l}\text { A. Gehrt s/n: } \\
\text { SP } 4559\end{array}$ \\
\hline Iridaceae & $\begin{array}{l}\text { Neomarica imbricata } \\
\text { (Hand.-Mazz.) Sprague }\end{array}$ & Herb & Ter & Auto & Forest & Auto & $\mathrm{NE}$ & $\begin{array}{l}\text { A. Gehrt s/n: } \\
\text { SP } 4659\end{array}$ \\
\hline
\end{tabular}


Table 1. Continued...

\begin{tabular}{|c|c|c|c|c|c|c|c|c|}
\hline Family & Species & Life-form & Substrate & Origin & Habitat & Syndrome & Status & Voucher \\
\hline Lamiaceae & $\begin{array}{c}\text { Mesosphaerum sidifolium } \\
\text { (L'Hérit.) Harley \& J.F.B. } \\
\text { Pastore }\end{array}$ & $\begin{array}{l}\text { Herb, } \\
\text { Subshrub }\end{array}$ & Ter & Auto & Forest edge & Zoo & $\mathrm{NE}$ & $\begin{array}{l}\text { V.C. Souza 11065: } \\
\quad \text { ESA } 26141\end{array}$ \\
\hline \multirow[t]{2}{*}{ Malvaceae } & Sida planicaulis Cav.* & $\begin{array}{l}\text { Shrub, } \\
\text { Subshrub }\end{array}$ & Ter & Auto & $\begin{array}{l}\text { Forest edge, } \\
\text { Anthropogenic } \\
\text { vegetation }\end{array}$ & Zoo & $\mathrm{NE}$ & $\begin{array}{l}\text { A.M. Magalhães 40: } \\
\text { RB } 636577\end{array}$ \\
\hline & Sida rhombifolia L.* & Herb & Ter & Auto & $\begin{array}{l}\text { Forest edge, } \\
\text { Anthropogenic } \\
\text { vegetation }\end{array}$ & Zoo & $\mathrm{NE}$ & $\begin{array}{l}\text { A.M. Magalhães 52: } \\
\text { RB } 636583\end{array}$ \\
\hline Marantaceae & $\begin{array}{c}\text { Maranta divaricata } \\
\text { Roscoe }\end{array}$ & Herb & Ter & Auto & Forest & Auto & $\mathrm{NE}$ & $\begin{array}{l}\text { A.M. Magalhães 97: } \\
\text { RB } 636612\end{array}$ \\
\hline Meliaceae & Trichilia casaretti C. DC. & Tree & Ter & Auto & Forest & Zoo & $\mathrm{LC}[\mathrm{VU}]$ & $\begin{array}{l}\text { D.F. Bertani QT-96: } \\
\text { RB } 552254\end{array}$ \\
\hline Musaceae & Musa paradisiaca L. & Herb & Ter & Allo & Banana plantation & Sterile & $\mathrm{NE}$ & Not collected \\
\hline \multirow[t]{2}{*}{ Myrtaceae } & $\begin{array}{c}\text { Eugenia astringens } \\
\text { Cambess. }\end{array}$ & Tree & Ter & Auto & Forest & Zoo & $\mathrm{NE}$ & $\begin{array}{l}\text { D.F. Bertani QP-80: } \\
\text { RB } 552194\end{array}$ \\
\hline & $\begin{array}{c}\text { Eugenia sulcata Spring } \\
\text { ex Mart. }\end{array}$ & Tree & Ter & Auto & Forest & Zoo & $\mathrm{NE}$ & $\begin{array}{l}\text { A. Gehrt s/n: } \\
\text { SP } 4528\end{array}$ \\
\hline Nyctaginaceae & $\begin{array}{c}\text { Guapira opposita (Vell.) } \\
\text { Reitz }\end{array}$ & Shrub, Tree & Ter & Auto & $\begin{array}{c}\text { Forest, } \\
\text { Anthropogenic } \\
\text { vegetation }\end{array}$ & Zoo & $\mathrm{NE}$ & $\begin{array}{l}\text { A.M. Magalhães 60: } \\
\text { RB } 636586\end{array}$ \\
\hline \multirow[t]{8}{*}{ Orchidaceae } & $\begin{array}{c}\text { Acianthera saundersiana } \\
\text { (Rchb. f.) Pridgeon \& } \\
\text { M.W. Chase }\end{array}$ & Herb & Epi & Auto & No information & Anemo & $\mathrm{NE}[\mathrm{LC}]$ & $\begin{array}{l}\text { A. Gehrt s/n: SP } \\
5452\end{array}$ \\
\hline & Cattleya intermedia Grah. & Herb & Epi, Rup & Auto & No information & Anemo & $\begin{array}{c}\mathrm{VU} \\
\{\mathrm{VU}\}\end{array}$ & $\begin{array}{l}\text { A. Gehrt s/n: } \\
\text { SP } 8146\end{array}$ \\
\hline & $\begin{array}{c}\text { Cyclopogon bicolor (Ker- } \\
\text { Gaw.) Schltr. }\end{array}$ & Herb & Ter & Auto & Forest & Anemo & $\mathrm{NE}$ & $\begin{array}{c}\text { G.O. Joaquim Jr. 23: } \\
\text { ESA } 33124\end{array}$ \\
\hline & $\begin{array}{c}\text { Eltroplectris calcarata } \\
\text { (Sw.) Garay \& Sweet }\end{array}$ & Herb & Ter & Auto & Forest & Anemo & $\mathrm{LC}$ & $\begin{array}{c}\text { G.O. Joaquim Jr. 24: } \\
\text { ESA } 33125\end{array}$ \\
\hline & $\begin{array}{l}\text { Epidendrum densiflorum } \\
\text { Hook. }\end{array}$ & Herb & Epi & Auto & Forest & Anemo & $\mathrm{NE}$ & $\begin{array}{l}\text { A. Gehrt s/n: } \\
\text { SP } 4621\end{array}$ \\
\hline & $\begin{array}{c}\text { Epidendrum fulgens } \\
\text { Brongn. }\end{array}$ & Herb & Rup, Ter & Auto & $\begin{array}{l}\text { Rock outcrop } \\
\text { vegetation, } \\
\text { Anthropogenic } \\
\text { vegetation }\end{array}$ & Anemo & $\mathrm{NE}$ & $\begin{array}{l}\text { A. Amaral s/n: } \\
\text { SP } 3967\end{array}$ \\
\hline & $\begin{array}{c}\text { Mesadenella cuspidata } \\
\text { (Lindl.) Garay }\end{array}$ & Herb & Ter & Auto & Forest & Anemo & $\mathrm{NE}$ & $\begin{array}{l}\text { V.C. Souza 11042: } \\
\text { ESA } 27425\end{array}$ \\
\hline & $\begin{array}{l}\text { Oeceoclades maculata } \\
\text { (Lindl.) Lindl. }{ }^{\dagger}\end{array}$ & Herb & Ter & Allo & Forest & Anemo & $\mathrm{NE}[\mathrm{LC}]$ & $\begin{array}{l}\text { V.C. Souza 11040: } \\
\text { ESA } 27423\end{array}$ \\
\hline Passifloraceae & Passiflora suberosa L.* & Climber & Ter & Auto & Forest edge & Zoo & $\mathrm{NE}$ & $\begin{array}{l}\text { V.C. Souza 11025: } \\
\text { RB } 482678\end{array}$ \\
\hline \multirow[t]{2}{*}{ Phytolaccaceae } & $\begin{array}{l}\text { Gallesia integrifolia } \\
\text { (Spreng.) Harms }\end{array}$ & Tree & Ter & Auto & Forest & Anemo & $\mathrm{NE}$ & $\begin{array}{l}\text { A.M. Magalhães 50: } \\
\text { RB } 636581\end{array}$ \\
\hline & Rivina humilis L. & Herb & Ter & Allo & $\begin{array}{c}\text { Forest, Forest edge, } \\
\text { Anthropogenic } \\
\text { vegetation }\end{array}$ & Zoo & $\mathrm{NE}$ & $\begin{array}{c}\text { A.M. Magalhães 67: } \\
\text { RB } 636592\end{array}$ \\
\hline Piperaceae & $\begin{array}{c}\text { Peperomia glabella (Sw.) } \\
\text { A. Dietr. }\end{array}$ & Herb & Epi & Auto & No information & Zoo & $\mathrm{NE}$ & $\begin{array}{l}\text { L.E. Mello Filho 1979: } \\
\text { NY } 558881\end{array}$ \\
\hline Plantaginaceae & Scoparia dulcis L. & $\begin{array}{l}\text { Herb, } \\
\text { Subshrub }\end{array}$ & Ter & Auto & $\begin{array}{c}\text { Rock outcrop } \\
\text { vegetation }\end{array}$ & Anemo & $\mathrm{NE}$ & $\begin{array}{l}\text { V.C. Souza 11084: } \\
\text { ESA } 27112\end{array}$ \\
\hline Plumbaginaceae & Plumbago scandens L. & Subshrub & Ter & Auto & $\begin{array}{c}\text { Anthropogenic } \\
\text { vegetation }\end{array}$ & Zoo & $\mathrm{NE}$ & $\begin{array}{l}\text { A.M. Magalhães 36: } \\
\text { RB } 636574\end{array}$ \\
\hline \multirow[t]{5}{*}{ Poaceae } & $\begin{array}{c}\text { Digitaria ciliaris (Retz.) } \\
\text { Koeler* }\end{array}$ & Herb & Ter & Allo & $\begin{array}{l}\text { Anthropogenic } \\
\text { vegetation }\end{array}$ & Anemo, Zoo & $\mathrm{NE}$ & $\begin{array}{l}\text { A.M. Magalhães 93: } \\
\text { RB } 636608\end{array}$ \\
\hline & $\begin{array}{c}\text { Digitaria insularis (L.) } \\
\text { Fedde* }\end{array}$ & Herb & Ter & Allo & $\begin{array}{c}\text { Anthropogenic } \\
\text { vegetation }\end{array}$ & Anemo, Zoo & $\mathrm{NE}$ & $\begin{array}{l}\text { A.M. Magalhães 90: } \\
\text { RB } 636605\end{array}$ \\
\hline & $\begin{array}{c}\text { Eleusine indica (L.) } \\
\text { Gaertn.* }\end{array}$ & Herb & Ter & Allo & $\begin{array}{l}\text { Anthropogenic } \\
\text { vegetation }\end{array}$ & Zoo & $\mathrm{NE}[\mathrm{LC}]$ & $\begin{array}{l}\text { A.M. Magalhães 87: } \\
\text { RB } 636603\end{array}$ \\
\hline & $\begin{array}{l}\text { Lasiacis ligulata Hitchc. } \\
\text { \& Chase }\end{array}$ & Herb & Ter & Auto & Forest & Zoo & $\mathrm{NE}$ & $\begin{array}{c}\text { V.C. Souza 11062: } \\
\text { ESA } 26138\end{array}$ \\
\hline & $\begin{array}{c}\text { Melinis minutiflora P. } \\
\text { Beauv. }{ }^{\dagger}\end{array}$ & Herb & Ter & Allo & $\begin{array}{c}\text { Anthropogenic } \\
\text { vegetation }\end{array}$ & Anemo & $\mathrm{NE}$ & $\begin{array}{c}\text { F.T. Farah 9: } \\
\text { ESA } 39563\end{array}$ \\
\hline
\end{tabular}


Table 1. Continued...

\begin{tabular}{|c|c|c|c|c|c|c|c|c|}
\hline Family & Species & Life-form & Substrate & Origin & Habitat & Syndrome & Status & Voucher \\
\hline & Paspalum distichum L. & Herb & Ter & Auto & $\begin{array}{c}\text { Anthropogenic } \\
\text { vegetation }\end{array}$ & Hydro, Zoo & $\mathrm{NE}[\mathrm{LC}]$ & $\begin{array}{c}\text { G.O. Joaquim Jr. 44: } \\
\text { ESA } 33136\end{array}$ \\
\hline & Paspalum virgatum L.* & Herb & Ter & Auto & $\begin{array}{c}\text { Anthropogenic } \\
\text { vegetation }\end{array}$ & Auto & $\mathrm{NE}$ & $\begin{array}{l}\text { A.M. Magalhães 91: } \\
\text { RB } 636606\end{array}$ \\
\hline & $\begin{array}{l}\text { Sporobolus virginicus } \\
\text { (L.) Kunth }\end{array}$ & Herb & Ter & Auto & $\begin{array}{l}\text { Rock outcrop } \\
\text { vegetation }\end{array}$ & Anemo & $\mathrm{NE}$ & $\begin{array}{l}\text { A.M. Magalhães 92: } \\
\text { RB } 636607\end{array}$ \\
\hline & $\begin{array}{c}\text { Stenotaphrum } \\
\text { secundatum (Walter) } \\
\text { Kuntze }\end{array}$ & Herb & Ter & Auto & $\begin{array}{l}\text { Rock outcrop } \\
\text { vegetation, } \\
\text { Anthropogenic } \\
\text { vegetation }\end{array}$ & Hydro, Zoo & $\mathrm{NE}$ & $\begin{array}{c}\text { A.M. Magalhães 86: } \\
\text { RB } 636602\end{array}$ \\
\hline Polygonaceae & $\begin{array}{c}\text { Ruprechtia laurifolia } \\
\text { (Cham. \& Schltdl.) A.C. } \\
\text { Meyer }\end{array}$ & $\begin{array}{l}\text { Tree, } \\
\text { Climber }\end{array}$ & Ter & Auto & No information & Anemo & $\mathrm{NE}$ & $\begin{array}{l}\text { A. Gehrt s/n: } \\
\text { UEC } 80555\end{array}$ \\
\hline \multirow[t]{2}{*}{ Portulacaceae } & Portulaca oleracea L. & Herb & Ter & Auto & $\begin{array}{l}\text { Rock outcrop } \\
\text { vegetation }\end{array}$ & Auto & $\mathrm{NE}$ & $\begin{array}{c}\text { A.M. Magalhães 73: } \\
\text { RB } 636596\end{array}$ \\
\hline & $\begin{array}{l}\text { Talinum paniculatum } \\
\text { (Jacq.) Gaertn. }\end{array}$ & Herb & Rup, Ter & Auto & $\begin{array}{l}\text { Rock outcrop } \\
\text { vegetation }\end{array}$ & Auto & $\mathrm{NE}$ & $\begin{array}{c}\text { A.M. Magalhães 100: } \\
\text { RB } 636614\end{array}$ \\
\hline Primulaceae & $\begin{array}{l}\text { Myrsine guianensis } \\
\text { (Aubl.) Kuntze }\end{array}$ & Shrub, Tree & Ter & Auto & Forest & Zoo & $\mathrm{NE}$ & $\begin{array}{c}\text { D.F. Bertani QT-2: } \\
\text { RB } 560747\end{array}$ \\
\hline Rubiaceae & $\begin{array}{l}\text { Chiococca alba (L.) } \\
\text { Hitchc. }\end{array}$ & Shrub & Ter & Auto & $\begin{array}{l}\text { Forest edge, } \\
\text { Anthropogenic } \\
\text { vegetation }\end{array}$ & Zoo & $\mathrm{NE}[\mathrm{LC}]$ & $\begin{array}{c}\text { A.M. Magalhães 61: } \\
\text { RB } 636587\end{array}$ \\
\hline \multirow{4}{*}{ Sapindaceae } & $\begin{array}{c}\text { Rudgea minor (Cham.) } \\
\text { Standl. }\end{array}$ & Shrub, Tree & Ter & Auto & Forest & Zoo & $\mathrm{LC}$ & $\begin{array}{c}\text { A.M. Magalhães 71: } \\
\text { RB } 636595\end{array}$ \\
\hline & $\begin{array}{l}\text { Cardiospermum } \\
\text { halicacabum L.* }\end{array}$ & $\begin{array}{c}\text { Herb, } \\
\text { Climber }\end{array}$ & Ter & Auto & $\begin{array}{c}\text { Anthropogenic } \\
\text { vegetation }\end{array}$ & Zoo & $\mathrm{NE}$ & $\begin{array}{l}\text { A.M. Magalhães 102: } \\
\text { RB } 636616\end{array}$ \\
\hline & $\begin{array}{c}\text { Cupania oblongifolia } \\
\text { Mart. }\end{array}$ & Tree & Ter & Auto & Forest & Zoo & $\mathrm{NE}$ & $\begin{array}{l}\text { D.F. Bertani QT-23: } \\
\text { RB } 551934\end{array}$ \\
\hline & $\begin{array}{c}\text { Urvillea triphylla (Vell.) } \\
\text { Radlk. }\end{array}$ & Climber & Ter & Auto & Forest edge & Anemo & $\mathrm{NE}$ & $\begin{array}{c}\text { V.C. Souza 11075: } \\
\text { ESA } 27104\end{array}$ \\
\hline Sapotaceae & $\begin{array}{l}\text { Sideroxylon obtusifolium } \\
\text { (Roem. \& Schult.) T.D. } \\
\text { Penn. }\end{array}$ & Shrub, Tree & Ter & Auto & Forest & Zoo & $\mathrm{LC}$ & $\begin{array}{l}\text { A.M. Magalhães s/n: } \\
\text { RB } 637081\end{array}$ \\
\hline Urticaceae & Pilea pubescens Liebm. & Herb & Ter & Auto & No information & Auto & $\mathrm{NE}$ & $\begin{array}{c}\text { A.R. Duarte 56: } \\
\text { ESA } 49651\end{array}$ \\
\hline Vitaceae & $\begin{array}{l}\text { Cissus verticillata (L.) } \\
\text { Nicolson \& C.E. Jarvis }\end{array}$ & Climber & Ter & Auto & Forest edge & Zoo & $\mathrm{NE}$ & $\begin{array}{l}\text { A.M. Magalhães 101: } \\
\text { RB } 636615\end{array}$ \\
\hline
\end{tabular}

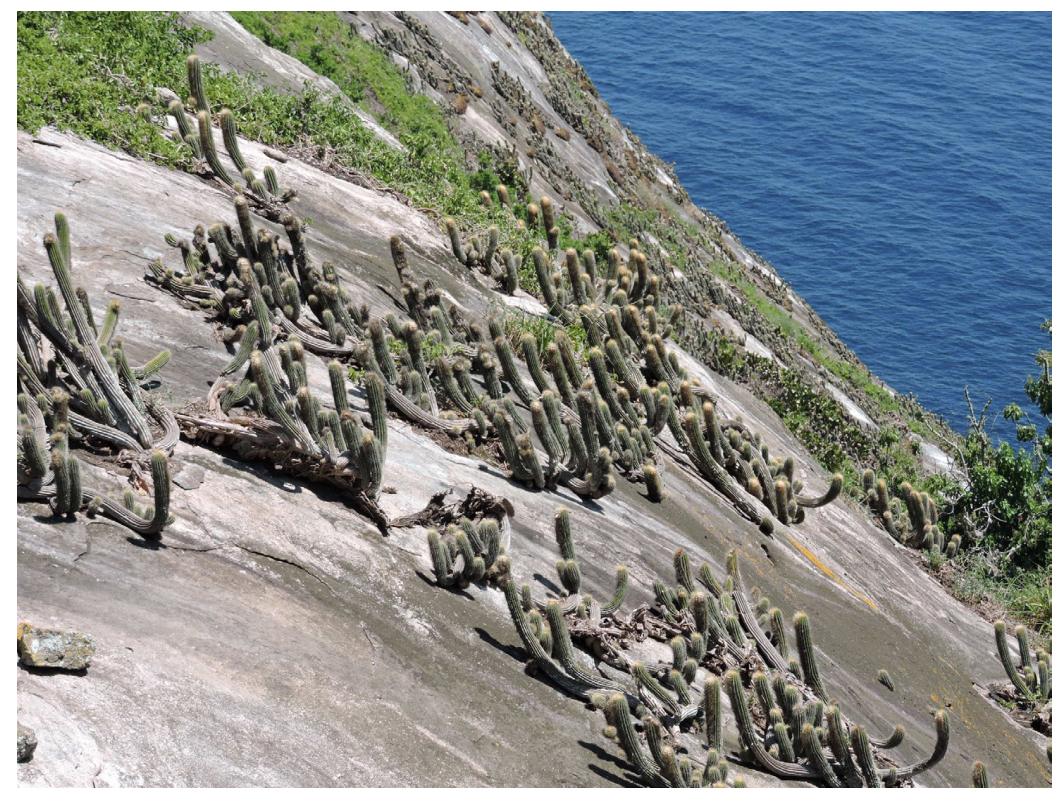

Figure 3. Rock outcrop vegetation on a steep cliff, with a large population of Coleocephalocereus fluminensis, Queimada Grande Island, São Paulo, southeastern Brazil. Photo by A.M. Magalhães. 
Table 2. Number of plant species on islands off the coast of eastern and southeastern Brazil.

\begin{tabular}{|c|c|c|c|c|c|c|}
\hline Site/Code & Coordinates & Phytophysiognomies & $\begin{array}{l}\text { Approximate } \\
\text { area (ha)/ } \\
\text { Maximum } \\
\text { altitude (m) }\end{array}$ & $\begin{array}{l}\text { Distance to } \\
\text { mainland } \\
(\mathbf{k m})\end{array}$ & $\begin{array}{l}\text { Number of } \\
\text { species }\end{array}$ & Reference \\
\hline $\begin{array}{l}\text { Queimada Grande } \\
\text { Island, Peruíbe, São } \\
\text { Paulo (QGI) }\end{array}$ & $\begin{array}{l}24^{\circ} 29^{\prime} 10^{\prime \prime} \mathrm{S}, 46^{\circ} 40^{\prime} 30^{\prime \prime} \mathrm{W} \\
\text { (center point) }\end{array}$ & $\begin{array}{l}\text { Dense Ombrophilous Forest, rock } \\
\text { outcrop vegetation, anthropogenic } \\
\text { vegetation }\end{array}$ & $57 / 210$ & 33.2 & $\begin{array}{c}125 \\
\text { (vascular } \\
\text { plants) }\end{array}$ & This study \\
\hline $\begin{array}{l}\text { Abrolhos Marine } \\
\text { National Park, } \\
\text { Caravelas, Bahia } \\
\text { (ABR)* }\end{array}$ & $\begin{array}{l}17^{\circ} 57^{\prime} 35^{\prime \prime}-17^{\circ} 58^{\prime} 56^{\prime \prime} \mathrm{S}, \\
38^{\circ} 41^{\prime} 27^{\prime \prime}-38^{\circ} 42^{\prime} 56^{\prime \prime} \mathrm{W}\end{array}$ & Rock outcrop vegetation, restinga & $77 / 36$ & 65 & $\begin{array}{c}40 \\
\text { (angiosperms) }\end{array}$ & Kemenes (2003) \\
\hline $\begin{array}{l}\text { Franceses Island, } \\
\text { Itapemirim, Espírito } \\
\text { Santo (FRA) }\end{array}$ & $\begin{array}{c}20^{\circ} 55^{\prime} 36^{\prime \prime} \mathrm{S}, 40^{\circ} 45^{\prime} 15^{\prime \prime} \mathrm{W} \\
\text { (center point) }\end{array}$ & $\begin{array}{l}\text { Dense Ombrophilous Forest, } \\
\text { rock outcrop vegetation, restinga, } \\
\text { mangrove swamps, anthropogenic } \\
\text { vegetation }\end{array}$ & $16 / 36$ & 3.5 & $\begin{array}{c}123 \\
\text { (angiosperms) }\end{array}$ & Ferreira et al. (2007) \\
\hline $\begin{array}{l}\text { Cagarras Islands } \\
\text { Natural Monument, } \\
\text { Rio de Janeiro, Rio de } \\
\text { Janeiro }(\mathrm{CAG})^{* *}\end{array}$ & $\begin{array}{l}23^{\circ} 01^{\prime} 30^{\prime \prime}-23^{\circ} 04^{\prime} 32^{\prime \prime} \mathrm{S}, \\
43^{\circ} 11^{\prime} 23^{\prime \prime}-43^{\circ} 12^{\prime} 32^{\prime \prime} \mathrm{W}\end{array}$ & $\begin{array}{l}\text { Dense Ombrophilous Forest, rock } \\
\text { outcrop vegetation, anthropogenic } \\
\text { vegetation }\end{array}$ & $79 / 240$ & $3.8-8.6$ & $\begin{array}{l}169 \\
\text { (vascular } \\
\text { plants) }\end{array}$ & Bovini et al. (2014) \\
\hline $\begin{array}{l}\text { Grande Island, Angra } \\
\text { dos Reis, Rio de } \\
\text { Janeiro (GRA) }\end{array}$ & $\begin{array}{l}23^{\circ} 04^{\prime} 30^{\prime \prime}-23^{\circ} 13^{\prime} 40^{\prime \prime} \mathrm{S}, \\
44^{\circ} 05^{\prime} 26^{\prime \prime}-44^{\circ} 22^{\prime} 43^{\prime \prime} \mathrm{W}\end{array}$ & $\begin{array}{l}\text { Dense Ombrophilous Forest, } \\
\text { rock outcrop vegetation, restinga, } \\
\text { mangrove swamps, anthropogenic } \\
\text { vegetation }\end{array}$ & $19,300 / 1,011$ & 3.1 & $\begin{array}{l}795 \\
\text { (vascular } \\
\text { plants) }\end{array}$ & Callado et al. (2009) \\
\hline $\begin{array}{c}\text { Cardoso Island, } \\
\text { Cananéia, São Paulo } \\
\text { (CAR) }\end{array}$ & $\begin{array}{l}25^{\circ} 03^{\prime} 05^{\prime \prime}-25^{\circ} 18^{\prime} 18^{\prime \prime} \mathrm{S}, \\
47^{\circ} 53^{\prime} 48^{\prime \prime}-48^{\circ} 05^{\prime} 42^{\prime \prime} \mathrm{W}\end{array}$ & $\begin{array}{l}\text { Dense Ombrophilous Forest, } \\
\text { rock outcrop vegetation, restinga, } \\
\text { mangrove swamps, anthropogenic } \\
\text { vegetation }\end{array}$ & $22,500 / 840$ & $<1$ & $\begin{array}{c}985 \\
\text { (angiosperms) }\end{array}$ & Barros et al. (1991) \\
\hline $\begin{array}{c}\text { Mel Island, } \\
\text { Paranaguá, Paraná } \\
\text { (MEL) }\end{array}$ & $\begin{array}{l}25^{\circ} 29^{\prime} 00^{\prime \prime}-25^{\circ} 34^{\prime} 32^{\prime \prime} \mathrm{S}, \\
48^{\circ} 17^{\prime} 15^{\prime \prime}-48^{\circ} 23^{\prime} 16^{\prime \prime} \mathrm{W}\end{array}$ & $\begin{array}{l}\text { Dense Ombrophilous Forest, } \\
\text { rock outcrop vegetation, restinga, } \\
\text { mangrove swamps, anthropogenic } \\
\text { vegetation }\end{array}$ & $2,894 / 148$ & 2.8 & $\begin{array}{l}\sim 504 \\
\text { (vascular } \\
\text { plants) }\end{array}$ & $\begin{array}{c}\text { Kersten \& Silva (2005) } \\
\text { Kozera \& Rodrigues } \\
\text { (2005); Marques } \\
\text { \& Oliveira (2005); } \\
\text { Salino et al. (2005) }\end{array}$ \\
\hline
\end{tabular}

* The survey included the five islands that make up ABR: Guarita, Redonda, Santa Barbara, Siriba and Sueste.

** The survey included the four largest islands at CAG: Cagarra, Comprida, Palmas and Redonda.

\section{Discussion}

The small islands $(<100 \mathrm{ha})$ along the eastern and southeastern coast of Brazil have very non-diverse floras (Kemenes 2003, Ferreira et al. 2007, Bovini et al. 2014) related to their insular conditions, sizes, habitat restrictions, steep topographies, incipient soils, and use histories (see Lomolino 2000, Kreft et al. 2008). Species richness on QGI is lower than that on CAG (Bovini et al. 2014) and close to that on FRA (an island of only 16 ha; Ferreira et al. 2007) - which is apparently related to the distance of QGI from the coast, making the arrival and establishment of propagules more difficult. The very low richness on ABR (Kemenes 2003) can also be related to its greater distance from the coast.

Large islands generally have richer floras due to their great environmental heterogeneity (Barros et al. 1991, Kersten \& Silva 2005, Kozera \& Rodrigues 2005, Marques \& Oliveira 2005, Salino et al. 2005, Callado et al. 2009). These islands can be considered insular samples of the mainland Atlantic Forest complex, and they harbor many mainland plant communities, i.e., altitudinal gradients of dense ombrophilous forests and pioneer formations: restinga, rock outcrop vegetation, and mangrove swamps.

The vascular flora of QGI originated from the mainland Atlantic Forest complex, and the vast majority of its species (including most species associated with anthropically disturbed areas) are autochthonous to that southeastern Brazilian complex, occurring frequently in dense ombrophilous forests or restinga. This pattern was also reported for FRA (Ferreira et al. 2007) and CAG (Bovini et al. 2014). Some species are widespread on those three sets of islands (e.g., Sesuvium portulacastrum,
Temnadenia odorifera, Syagrus romanzoffiana, Cyrtocymura scorpioides, Cereus fernambucensis, Ipomoea cairica, Maranta divaricata, Guapira opposita, Sporobolus virginicus, Talinum paniculatum and Chiococca alba).

The Dense Ombrophilous Forest on QGI showed a very low richness of arboreal species $(S=17)$, contrasting with high richness often found in continental remnants of this formation (e.g., Scudeller et al. 2001). The island forest also showed an unusual oligarchic structure, with a predominance of Guapira opposita, Rudgea minor, and Aspidosperma australe (B.C. Kurtz et al., unpubl. data). QGI is essentially a small rocky outcrop with few areas with deep soils, which limits the growth, distribution, and diversity of tree species. This edaphic aspect could partly explain the low species richness of this life form and its distinct original physiognomy in contrast to continent forests or those of other large islands (e.g., Barros et al. 1991).

The tree species on QGI are mostly zoochoric, producing small fleshy fruits or arilate seeds consumed by the island's avifauna (e.g., Pineschi 1990, Lorenzi 1998, 2000, Passos \& Oliveira 2004). Due to the absence of frugivorous mammals on QGI (Marques et al. 2002), birds are apparently the main local dispersers of those diaspores, and several species are known to feed on the fruits of Guapira opposita, Myrsine guianensis, and Syagrus romanzoffiana, which fructify during long periods of time and represent an important food resource for the local avifauna (Montanhini 2010). Two omnivorous passerines that visit the island at different times of the year are the main dietary items of the endemic and critically endangered pit viper Bothrops insularis (Marques et al. 2012). 
The historical use of the island, especially during the period when the lighthouse was manually operated (1909-1925), strongly influenced the current vegetation cover of QGI and its flora. According to information obtained from the Brazilian Navy, the original Dense Ombrophilous Forest was reduced to construct the lighthouse and its support buildings, to establish a subsistence farm and, possibly, to create animal pasture. These changes were most likely made by cutting and burning the original vegetation (the latter was apparently a common practice that apparently gave rise to the island's name - 'Big Burn Island'; Bataus \& Reis 2011). Thus, there were intentional and unintentional introductions of several foreign plant species to QGI. This set of species seems to be well-established now on the island, competing with the original vegetation and preventing its full recovery. Although the largest area of anthropogenic vegetation (located near the lighthouse) has not expanded over the last 10 years, its natural recovery appears to be extremely slow (B.C. Kurtz et al., unpubl. data). Additionally, many of the species recorded by A. Gehrt in the early 1920's were not encountered during our more recent expeditions.

The flora of QGI can be further analyzed by considering the composition of its life forms. The number of tree species $(S=17)$ corresponds to roughly half the number of climbers $(S=31)$, a group that is widely considered an indicator of disturbance in tropical forests (Gerwing 2001; Laurance et al. 2001). The herbaceous flora $(S=52)$ is the richest among the various life forms on QGI, with several species occurring exclusively in the anthropogenic vegetation. Additionally, although the mainland Atlantic Forest shows very high vascular hemiepiphytic/epiphytic species richness (2,256 species or 15.4\% of its vascular flora; Freitas et al. 2016), our surveys showed a low richness of this group of plants on the island, making it plausible to argue that the past uses of QGI were responsible for major changes in its already impoverished insular flora.

Of the two species considered invasive in the Atlantic Forest complex (Instituto Hórus 2017), molasses grass (Melinis minutiflora; 'capim gordura') is abundant in the anthropogenic vegetation sites on QGI. It has been observed that massive occurrences of this grass are related to a number of factors that prevent the regeneration of natural environments - such as changes in nutrient cycles, light availability, soil microclimate, and wind velocity. Also, high infestations of molasses grass directly interfere with natural regeneration by creating a thick layer of plant material that lends considerable competitive advantage over other species of the lower stratum, including seedlings and saplings (Barger et al. 2003; Martins et al. 2004). Oeceoclades maculata, on the other hand, does not currently appear to behave as invasive species on QGI.

The introduction and establishment of allochthonous species is not a problem exclusive to QGI. Many small islands distributed along the southeastern coast of Brazil are partially covered by alien species (pers. obs.). The islands that make up CAG (Bovini et al. 2014), for example, are partially covered by Megathyrsus maximus (Jacq.) B.K. Simon \& S.W.L. Jacobs, a grass of African origin, and $13 \%$ of the surface of FRA (Ferreira et al. 2007) is occupied by Leucaena leucocephala (Lam.) de Wit, an arboreal American legume. These two alien species have great invasive potential in the Atlantic Forest complex (Instituto Hórus 2017), and the introduction of invasive alien species has been highlighted as one of the main threats to island biota around the world (Kreft et al. 2008, Serafini et al. 2010).

As such, we recommend permanent and routine monitoring of the vegetation cover of QGI, using both high-resolution satellite images and field studies, to evaluate changes in areas covered by anthropogenic vegetation. Managers should consider controlling alien species, especially the invasive grass Melinis minutiflora, and planting seedlings of native species identified in this study. These activities will be essential to the in situ conservation of the critically endangered Bothrops insularis.

\section{Acknowledgments}

We would like to thank the AREI team of Queimada Pequena and Queimada Grande Islands, Airton Lourenço Júnior (in memoriam), André Eterovic, Carlos Roberto Abrahão, Marcelo Duarte, Otávio Marques, Ricardo Augusto Dias, and Vivian Mara Uhlig for logistical support and security during excursions to the Queimada Grande Island, FAPESP and CNPq for financial support, A.S.F. Vaz, C.G.P. Quinet, C. Sakuragui, C. Kameyama, C.M. Mynssen, C.N. Fraga, C.F.C. Sá, D. Zappi, D.N.S. Machado, E.G. Gonçalves, E. Jacques, H.C. Lima, I. Cordeiro, I. Rodrigues, M. Alves, M. Sobral, M.A.N. Coelho, M.A. Farinaccio, M.L. Kawasaki, M. Gomes, M.G. Bovini, M. Kirizawa, P. Leitman, R. Esteves, R.S. Rodrigues, R. Simão-Bianchini, V. Scalon, and W. Forster for their help in identifying the plant material and providing information about the species, as well as the referees for their constructive criticism of the manuscript.

\section{Author Contributions}

Bruno Coutinho Kurtz: substantial contribution in the concept and design of the study; contribution to data collection, analysis, interpretation, and manuscript preparation.

Vinicius Castro Souza: substantial contribution in the concept and design of the study; contribution to data collection, analysis, interpretation, and manuscript preparation.

Adriana Melo Magalhães: contribution to data collection, analysis, and interpretation.

Juliana de Paula-Souza: substantial contribution in the concept and design of the study; contribution to data collection, analysis, interpretation, and manuscript preparation.

Alexandre Romariz Duarte: contribution to data collection.

Gilberto Oliveira Joaquim-Jr.: contribution to data collection.

\section{Conflicts of interest}

The authors declare that they have no conflict of interest related to the publication of this manuscript.

\section{References}

BARGER, N.N., D'ANTONIO, C.M., GHNEIM, T. \& CUEVAS, E. 2003. Constraints to colonization and growth of the African grass, Melinis minutiflora, in a Venezuelan savanna. Plant Ecol. 167(1):31-43.

BARROS, F., MELO, M.M.R.F., CHIEA, S.A.C., KIRIZAWA, M., WANDERLEY, M.G.L. \& JUNG-MENDAÇOLLI, S.L. 1991. Caracterização geral da vegetação e listagem das espécies ocorrentes. In Flora fanerogâmica da Ilha do Cardoso (M.M.R.F. Melo, F. Barros, M.G.L. Wanderley, M. Kirizawa, S.L. JungMendaçolli \& S.A.C. Chiea, eds.). Instituto de Botânica, São Paulo, v.1, p.1-184.

BARROSO, G.M., MORIM, M.P., PEIXOTO, A.L. \& ICHASO, C.L.F. 1999. Frutos e sementes: morfologia aplicada à sistemática de dicotiledôneas. UFV, Viçosa.

BATAUS, Y.S.L. \& REIS, M.L. (orgs.). 2011. Plano de ação nacional para a conservação da herpetofauna insular ameaçada de extinção. Instituto Chico Mendes de Conservação da Biodiversidade, Brasília.

BOVINI, M.G., CARVALHO-OKANO, R.M. \& VIEIRA, M.F. 2001. Malvaceae A. Juss. no Parque Estadual do Rio Doce, Minas Gerais, Brasil. Rodriguésia 52(81):17-47.

BOVINI, M., FARIA, M., OLIVEIRA, R. \& KURTZ, B. 2013. Flora terrestre vascular: riqueza, biogeografia e vulnerabilidades. In História, pesquisa e biodiversidade do Monumento Natural das Ilhas Cagarras (F. Moraes, A. Bertoncini \& A. Aguiar, eds.). Museu Nacional, Rio de Janeiro, p.139-161.

BOVINI, M.G., FARIA, M., OLIVEIRA, R.R. \& KURTZ, B.C. 2014. Floristic diversity of the Cagarras Islands Natural Monument, Rio de Janeiro, Brazil. Check List 10(2):366-373. 
BRASIL. 2014. Portaria MMAn ${ }^{\circ} 444,17$ December 2014. Diário Oficial da União, edição 245, seção 1:121-126, 18 December 2014.

CALlADO, C.H., BARROS, A.A.M., RIBAS, L.A., ALBARELlO, N., GAGLIARDI, R. \& JASCONE, C.E.S. 2009. Flora e cobertura vegetal. In O ambiente da Ilha Grande (M. Bastos \& C.H. Callado, orgs.). UERJ, CEADS, Rio de Janeiro, p.91-161.

CAMPOS, J.S. \& MELLO-FILHO, L.E. 1966. Observações biológicas sobre a Ilha de Queimada Grande. Folha Med. 52(5):343-366.

CEPAGRI. 2017. Clima dos municípios paulistas. Centro de Pesquisas Meteorológicas e Climáticas Aplicadas à Agricultura. http://www.cpa.unicamp.br/outrasinformacoes/clima-dos-municipios-paulistas.html (last access in 22/09/2017)

CNCFLORA. 2017. Lista Vermelha. Centro Nacional de Conservação da Flora http://cncflora.jbrj.gov.br/portal/pt-br/listavermelha (last access in 09/04/2017)

FERREIRA, A.L., COUTINHO, B.R., PINHEIRO, H.T. \& THOMAZ, L.D. 2007. Composição florística e formações vegetais da Illha dos Franceses, Espírito Santo. Bol. Mus. Biol. Mello Leitão, Nova Sér. 22:25-44.

FERREIRA, P.P.A. 2009. O gênero Ipomoea L. (Convolvulaceae) no Rio Grande do Sul. Dissertação de mestrado, Universidade Federal do Rio Grande do Sul, Porto Alegre.

FERREIRA, P.P.A. \& MIOTTO, S.T.S. 2013. O gênero Merremia (Convolvulaceae) na região Sul do Brasil. Rodriguésia 64(3):635-646.

FLORA DO BRASIL 2020 EM CONSTRUÇÃO. 2016. Jardim Botânico do Rio de Janeiro. http://floradobrasil.jbrj.gov.br/ (last access in 05/08/2016)

FREITAS, L., SALINO, A., MENINI NETO, L., ALMEIDA, T.E., MORTARA, S.R., STEHMANN, J.R., AMORIM, A.M., GUIMARÃES, E.F., COELHO, M.N., ZANIN, A., FORZZA, R.C. 2016. A comprehensive checklist of vascular epiphytes of the Atlantic Forest reveals outstanding endemic rates. PhytoKeys 58:65-79.

GERWING, J.J. 2001. Testing liana cutting and controlled burning as silvicultura treatments for a logged forest in the eastern Amazon. J. Appl. Ecol. 38(6):1264-1276.

GOMES-COSTA, G.A. \& ALVES, M. 2012. Flora da Usina São José, Igarassu, Pernambuco: Cucurbitaceae. Rodriguésia 63(4):817-829.

GUIMARÃES, M., MUNGUÍA-STEYER, R., DOHERTY JR, P.F., MARTINS, M. \& SAWAYA, R.J. 2014. Population dynamics of the critically endangered golden lancehead pitviper, Bothrops insularis: stability or decline? PLoS ONE 9(4):e95203.

IBGE. 2012. Manual técnico da vegetação brasileira. 2 ed. IBGE, Rio de Janeiro.

ICHASO, C.L.F. 1980. Morfologia das sementes de 35 gêneros de Scrophulariaceae do Brasil - sua aplicação à sistemática desta família. Rodriguésia 32(53):33-107.

INCT. 2016. Herbário virtual da flora e dos fungos. Instituto Nacional de Ciência e Tecnologia. http://inct.splink.org.br (last access in 05/08/2016)

INSTITUTO HÓRUS. 2017. Base de dados nacional de espécies exóticas invasoras I3N Brasil. Instituto Hórus de Desenvolvimento e Conservação Ambiental http://i3n.institutohorus.org.br/www (last access in 22/01/2017)

IUCN. 2017. The IUCN red list of threatened species: version 2017-2. IUCN. http:// www.iucnredlist.org (last access in 20/09/2017)

KEMENES, A. 2003. Distribuição espacial da flora terrestre fanerogâmica do Parque Nacional Marinho de Abrolhos, BA. Rev. Bras. Bot. 26(2):141-150.

KERSTEN, R.A. \& SILVA, S.M. 2005. Florística e estrutura de comunidades de epífitas vasculares da planície litorânea. In História natural e conservação da Ilha do Mel (M.C.M. Marques \& R.M. Britez, orgs.). Editora UFPR, Curitiba, p.125-143.

KOZERA, C. \& RODRIGUES, R.R. 2005. Floresta Ombrófila Densa Submontana: florística e estrutura do estrato inferior. In História natural e conservação da Ilha do Mel (M.C.M. Marques \& R.M. Britez, orgs.). Editora UFPR, Curitiba, p.103-123

KREFT, H., JETZ, W., MUTKE, J., KIER, G. \& BARTHLOTT, W. 2008. Global diversity of island floras from a macroecological perspective. Ecol. Lett. 11(2):116-127.

LACERDA, L.D., ARAUJO, D.S.D. \& MACIEL, N.C. 1993. Dry coastal ecosystems of the tropical Brazilian coast. In Dry coastal ecosystems: Africa, America, Asia and Oceania (E. van der Maarel, ed.). Elsevier, Amsterdam, p.477-493.

LAURANCE, W.F., PÉREZ-SALICRUP, D., DELAMÔNICA, P., FEARNSIDE, P.M., D’ANGELO, S., JEROZOLINSKI, A., POHL, L. \& LOVEJOY, T.E. 2001.
Rain forest fragmentation and the structure of Amazonian liana communities. Ecology 82(1):105-116.

LOMOLINO, M.V. 2000. A call for a new paradigm of island biogeography. Global Ecol. Biogeogr. 9(1):1-6.

LORENZI, H. 1998. Árvores brasileiras: manual de identificação e cultivo de plantas arbóreas nativas do Brasil. 2 ed. Editora Plantarum, Nova Odessa, v.2.

LORENZI, H. 2000. Plantas daninhas do Brasil: terrestres, aquáticas, parasitas e tóxicas. 3 ed. Instituto Plantarum, Nova Odessa.

MACARTHUR, R.H. \& WILSON, E.O. 1967. The theory of island biogeography. Princeton University Press, Princeton.

MANSANO, V.F., TOZZI, A.M.G.A. \& LEWIS, G.P. 2004. A revision of the South America genus Zollernia Wied-Neuw. \& Nees (Leguminosae, Papilionoideae, Swartzieae). Kew Bull. 59(4):497-520.

MARQUES, M.C.M. \& OLIVEIRA, P.E.A.M. 2005. Características reprodutivas das espécies vegetais da planície costeira. In História natural e conservação da Ilha do Mel (M.C.M. Marques \& R.M. Britez, orgs.). Editora UFPR, Curitiba, p.169-188.

MARQUES, O.A.V., MARTINS, M. \& SAZIMA, I. 2002. A jararaca da Ilha da Queimada Grande. Ciênc. Hoje 31(186):56-59.

MARQUES, O.A.V., MARTINS, M. \& SAZIMA, I. 2004. Bothrops insularis. The IUCN Red List of Threatened Species. Version 2015.3. http://www.iucnredlist. org (last access in 18/09/2015)

MARQUES, O.A.V., MARTINS, M., DEVELEY, P.F., MACARRÃO, A. \& SAZIMA, I. 2012. The golden lancehead Bothrops insularis (Serpentes: Viperidae) relies on two seasonally plentiful bird species visiting its island habitat. J. Nat. Hist. 46(13-14):885-895.

MARTINS, C.R., LEITE, L.L. \& HARIDASAN, M. 2004. Capim-gordura (Melinis minutiflora P. Beauv.), uma gramínea exótica que compromete a recuperação de áreas degradadas em unidades de conservação. Rev. Árvore 28(5):739-747.

MARTINS, M., SAWAYA, R.J. \& MARQUES, O.A.V. 2008. A first estimate of the population size of the critically endangered lancehead, Bothrops insularis. South Am. J. Herpetol. 3(2):168-174.

MEIRELLES, S.T., PIVELLO, V.R. \& JOLY, C.A. 1999. The vegetation of granite rock outcrops in Rio de Janeiro, Brazil, and the need for its protection. Environ. Conserv. 26(1):10-20

MONTANHINI, A.M. 2010. Avifauna da Ilha da Queimada Grande, SP: diversidade, estrutura trófica e sazonalidade. Dissertação de mestrado, Universidade Estadual Paulista Júlio de Mesquita Filho, São José do Rio Preto.

MORELLATO, P.C. \& LEITÃO-FILHO, H.F. 1996. Reproductive phenology of climbers in a southeastern Brazilian forest. Biotropica 28(2):180-191.

MORO, M.F., SOUZA, V.C., OLIVEIRA-FILHO, A.T., QUEIROZ, L.P., FRAGA, C.N., RODAL, M.J.N., ARAUJO, F.S. \& MARTINS, F.R. 2012. Alienígenas na sala: o que fazer com espécies exóticas em trabalhos de taxonomia, florística e fitossociologia? Acta Bot. Bras. 26(4):991-999.

OLIVEIRA-FILHO, A.T. \& FONTES, M.A.L. 2000. Patterns of floristic differentiation among Atlantic forests in southeastern Brazil and the influence of climate. Biotropica 32(4b):793-810.

PASSOS, L. \& OLIVEIRA, P.S. 2004. Interaction between ants and fruits of Guapira opposita (Nyctaginaceae) in a Brazilian sandy plain rainforest: ant effects on seeds and seedlings. Oecologia 139(3):376-382.

PENNINGTON, T.D. 1990. Sapotaceae, Flora Neotropica. The New York Botanical Garden, New York, v.52.

PENNINGTON, T.D., STYLES, B.T. \& TAYLOR, D.A.H. 1981. Meliaceae, Flora Neotropica. The New York Botanical Garden, New York, v.28.

PIJL, L. van der. 1982. Principles of dispersal in higher plants. 3 ed. SpringerVerlag, Berlin.

PINESCHI, R.B. 1990. Aves como dispersores de sete espécies de Rapanea (Myrsinaceae) no maciço do Itatiaia, estados do Rio de Janeiro e Minas Gerais. Ararajuba 1:73-78.

REIS, R.C.C. 2006. Palmeiras (Arecaceae) das restingas do estado do Rio de Janeiro, Brasil. Acta Bot. Bras. 20(3):501-512.

RODRIGUES, R.S., FLORES, A.S., MIOTTO, S.T.S. \& BAPTISTA, L.R.M. 2005 O gênero Senna (Leguminosae, Caesalpinioideae) no Rio Grande do Sul, Brasil. Acta Bot. Bras. 19(1):1-16. 
ROLIM, G.S., CAMARGO, M.B.P., LANIA, D.G. \& MORAES, J.F.L. 2007. Classificação climática de Köppen e de Thornthwaite e sua aplicabilidade na determinação de zonas agroclimáticas para o estado de São Paulo. Bragantia 66(4):711-720.

SALINO, A., SILVA, S.M., DITTRICH, V.A.O. \& BRITEZ, R.M. 2005. Flora pteridofítica. In História natural e conservação da Ilha do Mel (M.C.M. Marques \& R.M. Britez, orgs.). Editora UFPR, Curitiba, p.85-101.

SÃO PAULO. 2016. Resolução SMA n 57, 5 June 2016. Diário Oficial de São Paulo, edição 126 (120), seção 1:55-57, 30 June 2016.

SCARANO, F.R. 2002. Structure, function and floristic relationships of plant communities in stressful habitats marginal to the Brazilian Atlantic rainforest. Ann. Bot-London 90(4):517-524.

SCUDELLER, V.V., MARTINS, F.R. \& SHEPHERD, G.J. 2001. Distribution and abundance of arboreal species in the atlantic ombrophilous dense forest in Southeastern Brazil. Plant Ecol. 152(2):185-199.

SERAFINI, T.Z., FRANÇA, G.B. \& ANDRIGUETTO-FILHO, J.M. 2010. Ilhas oceânicas brasileiras: biodiversidade conhecida e sua relação com o histórico de uso e ocupação humana. Rev. Gest. Cost. Integr. 10(3):281-301.

SILVA, S.M. \& BRITEZ, R.M. 2005. A vegetação da planície costeira. In História natural e conservação da Ilha do Mel (M.C.M. Marques \& R.M. Britez, orgs.). Editora UFPR, Curitiba, p.49-84.

SILVA-LUZ, C.L., GOMES, C.G., PIRANI, J.R. \& HARLEY, R.M. 2012. Flora da Serra do Cipó, Minas Gerais: Lamiaceae. Bol. Bot. Univ. São Paulo 30(2):109-155.

SOARES NETO, R.L., MAGALHÃES, F.A.L., TABOSA, F.R.S., MORO, M.F., COSTA E SILVA, M.B. \& LOIOLA, M.I.B. 2014. Flora do Ceará, Brasil: Capparaceae. Rodriguésia 65(3):671-684.

SOUZA, M.C. \& MORIM, M.P. 2008. Subtribos Eugeniinae O. Berg e Myrtinae O. Berg (Myrtaceae) na restinga da Marambaia, RJ, Brasil. Acta Bot. Bras. 22(3):652-683.

TESSLER, M.G. \& GOYA, S.C. 2005. Processos costeiros condicionantes do litoral brasileiro. Rev. Dep. Geogr. Univ. São Paulo 17:11-23.

WANDERLEY, M.G.L., SHEPHERD, G.J. \& GIULIETTI, A.M. (coords.). 2001. Flora fanerogâmica do estado de São Paulo. FAPESP, HUCITEC, São Paulo, v.1.
WANDERLEY, M.G.L., SHEPHERD, G.J. \& GIULIETTI, A.M. (coords.). 2002. Flora fanerogâmica do estado de São Paulo. FAPESP, HUCITEC, São Paulo, v.2.

WANDERLEY, M.G.L., SHEPHERD, G.J., GIULIETTI, A.M. \& MELHEM, T.S. (coords.). 2003. Flora fanerogâmica do estado de São Paulo. FAPESP, RiMa, São Paulo, v.3.

WANDERLEY, M.G.L., SHEPHERD, G.J., GIULIETTI, A.M. \& MELHEM, T.S. (coords.). 2005. Flora fanerogâmica do estado de São Paulo. FAPESP, RiMa, São Paulo, v.4.

WANDERLEY, M.G.L., SHEPHERD, G.J., MELHEM, T.S. \& GIULIETTI, A.M. (coords.). 2007. Flora fanerogâmica do estado de São Paulo. Instituto de Botânica, São Paulo, v.5.

WANDERLEY, M.G.L., SHEPHERD, G.J., MELHEM, T.S., GIULIETTI, A.M. \& MARTINS, S.E. (coords.). 2009. Flora fanerogâmica do estado de São Paulo. Instituto de Botânica, FAPESP, São Paulo, v.6.

WANDERLEY, M.G.L., SHEPHERD, G.J., MELHEM, T.S., GIULIETTI, A.M. \& MARTINS, S.E. (coords.). 2012. Flora fanerogâmica do estado de São Paulo. Instituto de Botânica, São Paulo, v.7.

WANDERLEY, M.G.L., SHEPHERD, G.J., MELHEM, T.S., GIULIETTI, A.M. \& MARTINS, S.E. (coords.). 2016. Flora fanerogâmica do estado de São Paulo. Instituto de Botânica, São Paulo, v.8. http://botanica.sp.gov.br/ffesp_online/ (last access in 23/01/2017)

WÜSTER, W., DUARTE, M.R. \& SALOMÃO, M.G. 2005. Morphological correlates of incipient arboreality and ornithophagy in island pitvipers, and the phylogenetic position of Bothrops insularis. J. Zool. 266(1):1-10.

Received: 23/03/2017

Revised: 25/09/2017

Accepted: 01/10/2017

Published online: 23/10/2017 